\title{
Controlled growth of plasmonic heterostructures and their applications
}

\author{
Yao Zhong ${ }^{1,2 \dagger}$, Song Ma ${ }^{1 \dagger}$, Kai Chen ${ }^{3}$, Peng-Fei Wang ${ }^{1}$, Yun-Hang Qiu ${ }^{1}$, Shan Liang ${ }^{4}$, Li Zhou ${ }^{1^{*}}$, \\ Yanwei Chen $^{2^{*}}$ and Qu-Quan Wang ${ }^{1,3^{*}}$
}

\begin{abstract}
Plasmonic nanocrystals with unique properties have been extensively studied in the past decades. A combination of plasmonic materials with other characteristic materials of metals and semiconductors leads to properties far beyond single-component materials and excellent performances in many optical-related applications. In this review, we summarize the recent advances in the controlled growth of plasmonic heterostructures with a specific composition, morphology, size, and structural symmetry. Plasmonenhanced properties of the heterostructures and their excellent performances in applications are also discussed. The synthesis strategies and the intriguing properties of the plasmonic heterostructures provide great opportunity for applications in plasmon-enhanced nonlinear optics, optical spectroscopy, photocatalysis, solar energy conversion, and so on.
\end{abstract}

Keywords: surface plasmon resonance, metal-semiconductor heterostructures, photocatalysis, nonlinear optical effects

\section{INTRODUCTION}

Surface plasmon resonance (SPR) strongly enhances local electromagnetic fields and various light-matter interactions, which are largely dependent on the size, shape, composition, and local environment [1-3]. Moreover, plasmonic heterocrystals, which consist of two nanomaterials with integrated multifunctionalities in single colloidal nanoparticles, may demonstrate better performance than their individual components [4]. In recent decades, great efforts have been devoted to developing synthetic methods for the growth of diverse colloidal heterocrystals with controlled morphologies and optimized performances; the abovementioned colloidal heterocrystals have prospective applications ranging from photocatalysis and solar energy conversion to bioimaging as well as quantum information processing [5-8].

For instance, the simplest plasmonic heterocrystals consisting of $\mathrm{Au}$ and $\mathrm{Ag}$ have the advantages of a large tunability of the plasmon resonances, including electric and magnetic modes as well as multipole resonances, which have better sensitivity and high stability for practical applications $[9,10]$. Heterocrystals consisting of a plasmonic metal (such as $\mathrm{Au}, \mathrm{Ag}, \mathrm{Cu}, \mathrm{Al}$ ) and a photocatalytic metal (Pt or Pd) have the integrated advantages of an antenna-reactor effect as well as a high catalytic activity on a high-index plane; therefore, they have largely enhanced photocatalytic efficiency [11,12]. Furthermore, metal-semiconductor heterocrystals not only combine plasmonic and excitonic advantages but also have very efficient excitation energy and charge transfer; therefore, they may demonstrate plasmon-exciton coupling and have better photonic and photoelectronic performances than individual metals and semiconductors, such as the prominently enhanced optical Stark effect [13], plasmonexciton Fano interference [14], reversed saturable absorption $[15,16]$, and enhanced photocatalytic activity [8]. Self-doped semiconductors, such as $\mathrm{Cu}_{2-x} \mathrm{~S}$ and $\mathrm{Cu}_{2-x} \mathrm{Se}$ nanocrystals, have strong plasmon resonances in the near-infrared (NIR) region; therefore, $\mathrm{Cu}_{2-x} \mathrm{~S} / \mathrm{Au}$ heterostructures demonstrate plasmon-plasmon coupling and have excellent performance in photothermal therapy [17-

${ }^{1}$ Key Laboratory of Artificial Micro- and Nano-structures of the Ministry of Education, Department of Physics, Wuhan University, Wuhan 430072, China

${ }^{2}$ Centre for Advanced Optoelectronic Functional Materials Research, Northeast Normal University, Changchun 130024, China

${ }^{3}$ The institute for Advanced Studies, Wuhan University, Wuhan 430072, China

${ }^{4}$ Department of Physics, Hunan Normal University, Changsha 410081, China

These authors contributed equally to this work.

* Corrsenponding authors (emails: qqwang@whu.edu.cn (Wang QQ); yanweichen@nenu.edu.cn (Chen Y); zhouli@whu.edu.cn (Zhou L)) 
21]. In recent years, plasmonic heterocrystals containing more than two components, such as (CdS-Pt)@Au [22] and $\left(\mathrm{Cu}_{2-x} \mathrm{~S}-\mathrm{CdS}\right) @ \mathrm{Au}$ [23], have been synthesized, which combine the advantages of an antenna-reactor effect and a semiconductor. Multichannel charge transfers in these complex heterostructures further improve their photonic, photocatalytic, and photovoltaic performances. The optimized morphology and component configuration of the heterostructures are largely dependent on the specific photonic and photocatalytic applications.

The main mechanisms of plasmonic enhancement effects of plasmon-semiconductor heterostructures are summarized in Scheme $1[4,8,24-26]$, and the plasmonenhanced photocatalytic performance is frequently discussed in the following sections. SPRs with intense absorption produce (1) large local field enhancement. The radiative decay of SPRs leads to (2) large light scattering. Nonradiative SPR damping occurs through electronelectron interactions, successively generating (5) hot electrons and (3) heat. The nonradiative decay of SPR can also occur via (4) plasmon resonance energy transfer. The processes of (1) and (2) can improve the light absorption of semiconductor nanocrystals. The processes of (4) and (5) can enrich the population of photoexcited carriers through plasmonic light harvesting. The plasmonic photothermal effect of (3) can accelerate catalytic reactions.

In this article, we review the syntheses of metal-metal and metal-semiconductor heterostructures and introduce their plasmonic as well as photocatalytic properties. The following four sections are related to the four kinds of heterostructures: i) metallic heterocrystals containing two plasmonic metals; ii) metallic heterocrystals with plasmonic and catalytic metals; iii) semiconductor-metal heterostructures with two components, including symmetric and asymmetric overgrowth of semiconductors on metal nanocrystals; and iv) semiconductor-metal heterostructures with three components, including plasmonic and catalytic metals on one semiconductor and two semiconductors on one plasmonic metal.

\section{METALLIC HETEROCRYSTALS WITH TWO PLASMONIC METALS}

Plasmonic metallic nanocrystals exhibit characteristic SPRs determined by their composition, shape, size, etc. The controlled growth of metallic nanocrystals is significantly important for obtaining SPRs with an expected wavelength and intensity or other intriguing properties. In addition, the integration of two plasmonic metals can lead to more interesting SPRs and more active chemical properties. The configuration of heteronanocrystals has

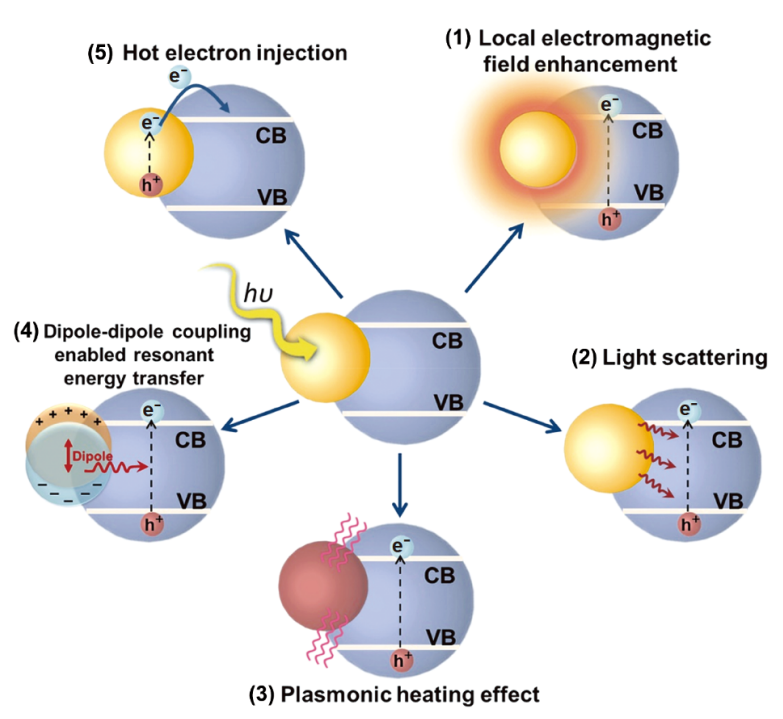

Scheme 1 For a plasmon-semiconductor heterostructure, the main plasmonic effects are shown as (1) a local field enhancement, (2) light scattering, (3) a photothermal effect, (4) plasmon resonance energy transfer, and (5) hot-electron transfer. Reprinted with permission from Ref. [24]. Copyright 2018, Elsevier.

several categories such as alloyed, core-shell, Janus and segment types. For the convenience of discussions, we use $\mathrm{AB}, \mathrm{B} @ \mathrm{~A}, \mathrm{~B} / \mathrm{A}$, and $\mathrm{C}-\mathrm{B}-\mathrm{A}$ to represent an $\mathrm{AB}$ alloy, a $\mathrm{B}$ shell on an A core, $B$ overgrown on the tip or one side of $\mathrm{A}$, and segmented heterostructures, respectively.

Kim et al. [27] prepared $\mathrm{AuCu}$ alloyed nanocrystals having different stoichiometric ratios of $\mathrm{Au}$-to- $\mathrm{Cu}(\mathrm{Au}$, $\mathrm{Au}_{3} \mathrm{Cu}, \mathrm{AuCu}, \mathrm{AuCu}_{3}$ and $\mathrm{Cu}$ ) with a uniform average size of approximately $10-11 \mathrm{~nm}$ via a co-reduction of metal precursors in an oil phase (Fig. 1a). Pure-phase Au and $\mathrm{Cu}$ nanoparticles exhibit characteristic SPR peaks at 523 and $570 \mathrm{~nm}$, respectively. Interestingly, as the content of $\mathrm{Cu}$ increases in the alloyed phase, the SPR peak of alloyed nanoparticles redshifts from the SPR peak of $\mathrm{Au}$ toward that of $\mathrm{Cu}$ with some broadening $[28,29](533 \mathrm{~nm}$ for $\mathrm{Au}_{3} \mathrm{Cu}, 539 \mathrm{~nm}$ for $\mathrm{AuCu}$ and $549 \mathrm{~nm}$ for $\mathrm{AuCu}_{3}$ ) (Fig. 1b). Similar results have also been observed in AuAg and $\mathrm{AgCu}$ alloyed nanocrystals [30-32]. Moreover, $\mathrm{AuCu}$ nanoparticle monolayer platforms with catalytically active surfaces were fabricated by a solvent evaporation-mediated self-assembly approach and the electrochemical $\mathrm{CO}_{2}$ reduction was evaluated [27]. $\mathrm{Au}_{3} \mathrm{Cu}$ nanoparticles exhibit the highest $\mathrm{CO}$ mass activity with a value of $-0.73 \mathrm{~V}$ vs. a reversible hydrogen electrode (RHE), while $\mathrm{Cu}$ nanoparticles exhibit the largest number in product types, for instance, many hydrocarbon species [33]. The results can be explained in terms of the electronic effect related to the binding of intermediates and the geometric effect 

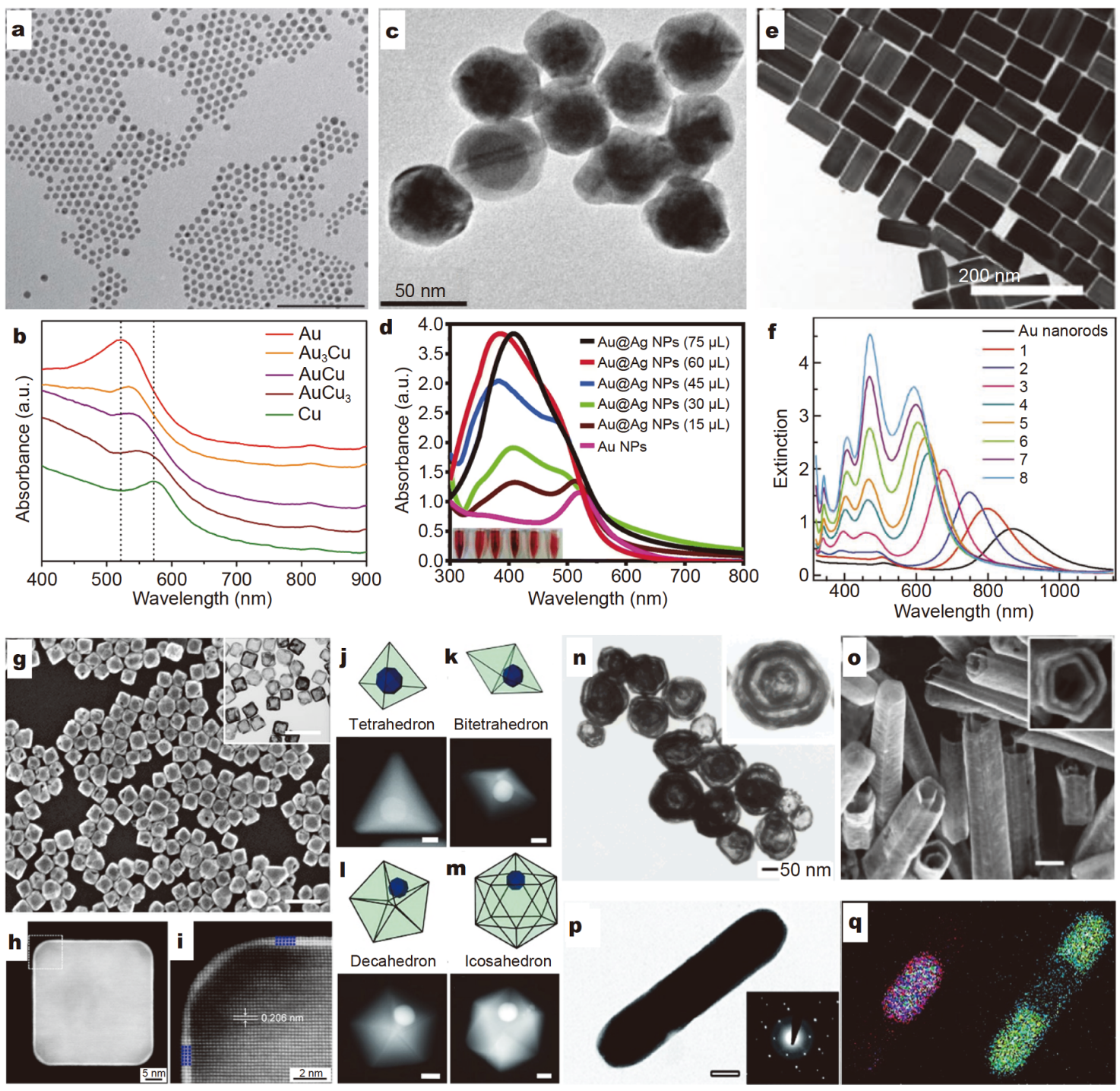

Figure 1 Heterostructures of plasmonic metals. (a, b) AuCu alloyed nanoparticles with tunable SPRs (scale bar, $100 \mathrm{~nm}$ ) by Kim et al. Reprinted with permission from Ref. [27]. Copyright 2014, Springer Nature. (c, d) Ag@Au core-shell nanoparticles with a tunable SPR in a range of 380-550 nm and used as colorimetric/Raman spectroscopic probes. Reprinted with permission from Ref. [35]. Copyright 2019, Springer Nature. (e, f) Ag@Au coreshell nanorods with four plasmon bands by Jiang et al. Reprinted with permission from Ref. [38]. Copyright 2012, John Wiley \& Sons. (g) AuAg alloyed nanocages prepared via a galvanic replacement reaction (scale bar, $100 \mathrm{~nm}$ ) by Skrabalak et al. Reprinted with permission from Ref. [49]. Copyright 2007, Springer Nature. (h, i) Au@Ag core-shell nanocubes with three atomic layers of Au, which improve the chemical stability for enhanced SERS activity by Yang et al. Reprinted with permission from Ref. [42]. Copyright 2014, American Chemical Society. (j-m) Ag@Au bimetallic particles with twinned structures grown from a pseudo-spherical single-crystalline Au seed via a plasmon-mediated reaction by Langille $e t$ al. Reprinted with permission from Ref. [46]. Copyright 2012, American Association for the Advancement of Science. AuAg alloyed hollow triplewalled nanoshells $(\mathrm{n})$ and double-walled (o) nanotubes (scale bar, $100 \mathrm{~nm}$ ) by Sun et al. (Reprinted with permission from Ref. [56]. Copyright 2004, American Chemical Society), and by Sun et al. (Reprinted with permission from Ref. [57]. Copyright 2004, John Wiley \& Sons). (p, q) Ag-Au-Ag heterometallic nanorods formed through anisotropic growth (scale bar, $50 \mathrm{~nm}$ ) by Seo et al. Reprinted with permission from Ref. [59]. Copyright 2008, American Chemical Society.

of the local atomic arrangement of the active site. More interestingly, $\mathrm{He}$ et al. [34] reported an aqueous phase route for the synthesis of pentacle-shaped $\mathrm{AuCu}$ alloy nanocrystals with fivefold twinning, which display three
SPR bands with one in the visible region and two in the NIR region. These $\mathrm{AuCu}$ nanocrystals demonstrated a notable photothermal effect toward killing 4T1 murine breast tumors and also demonstrated excellent catalytic 
activity.

Ag@Au core-shell nanoparticles can be synthesized by a reduction of silver nitrate with ascorbic acid (Fig. 1c, d) [35]; the above structure combines the advantages of the high refractive index sensitivity of silver and excellent stability of gold and demonstrates large tunability in plasmon resonances. They can be widely used in colorimetric chemical biosensing since the SPRs of gold and silver are both in the visible range. Hydrogen peroxide (generated from glucose catalyzed by glucose oxidase) directly oxidizes and gradually erodes the silver shell [36], leading to the solution color changing from brownish yellow to wine red without any indicator. This is more sensitive than other Ag nanoparticle-based materials that fade directly from yellow to colorless. The SERS intensity of this Ag@Au nanomaterial is very sensitive to the concentration of glucose in the presence of the oxidase [37]. The detection limit of this colorimetric and SERS dual-nanoprobe is $0.02 \mu \mathrm{mol} \mathrm{L} \mathrm{L}^{-1}$, which is 10 -fold lower than that of other Au nanoparticle-based materials. The dual-nanoprobe is also successfully used for the detection of serum samples. Moreover, Jiang et al. [38] reported the overgrowth of Ag shells on Au nanorods, and the obtained Ag@Au core-shell nanorods had four bands of plasmon resonance ranging from the visible to the NIR region (Fig. 1e, f). The lowest-energy peak belongs to the longitudinal dipolar plasmon mode, the second-lowestenergy peak is the transverse dipole plasmon mode, and the two highest-energy peaks can be ascribed to octupolar plasmon modes $[39,40]$. These different plasmon modes of the Ag@Au core-shell nanorods have advantages in various plasmon-based applications. In particular, the octupolar plasmon resonance has a higher $Q$ factor than the dipolar resonance, which is used for high-sensitivity sensors as well as the enhancement of second-order harmonic generation [41].

The synthesis approaches for preparing core-shell type heteronanocrystals with a precisely controlled dimension and morphology have been developed [42-46]. Yang et al. [42] reported a galvanic replacement-free deposition of $\mathrm{Au}$ on $\mathrm{Ag}$ nanocubes by introducing a fast reduction from adjusting the $\mathrm{pH}$ of the solution. An ultrathin Au shell of $0.6 \mathrm{~nm}$ thick with three atomic layers was able to deposit on the Ag nanocubes (Fig. 1h, i). The combination of excellent SERS activity from the high-quality SPRs in Ag and the chemical stability of Au were exhibited. Langille et al. [46] used spherical, cubic, and octahedral singlecrystalline gold nanoparticles as seeds and tracked the growth of multiple-twinned silver nanostructures through a light-induced and plasmon-mediated reaction
$[47,48]$. As shown in Fig. 1j-m, a pseudospherical singlecrystalline Au seed was observed to be coated by a singlecrystalline tetrahedron and even by a twinned bitetrahedron, 5-fold twinned decahedron, and 20-fold twinned icosahedron.

Bimetallic hollow nanostructures have attracted considerable attention due to their complex morphology and composition. Skrabalak et al. [49] developed a method using a galvanic replacement reaction between silver and chloroauric acid for preparing an abundance of AuAg alloyed hollow and porous nanostructures; moreover, the morphology is determined by the initiation of the $\mathrm{Ag}$ nanostructures. As shown in Fig. 1g, the uniform AuAg alloyed nanocages are prepared by using a template of high-quality Ag nanocubes. The galvanic replacement reaction provides a facile way to precisely tune the SPRs by adjusting the amount of $\mathrm{HAuCl}_{4}$ relative to that of the $\mathrm{Ag}$ templates [50-55]. The hollow metal nanostructures with multiple walls can be synthesized by coupling galvanic replacement with sequentially deposited templates, as the AuAg alloy nanoshells contain triple-walled and double-walled nanotubes as shown in Fig. 1n, o [56,57]. The advantages of the tunable SPRs extending to the NIR region, the large reactive surface with inner and outer surfaces, the porous surface and hollow interiors, and the tunable composition make these hollow and porous nanostructures attractive for various practical applications [52-55].

Many other heteronanocrystals combining two plasmonic metals with a symmetrical and an asymmetrical configuration were also reported [55-60]. Seo et al. $[58,59]$ reported the growth of $\mathrm{Ag}-\mathrm{Au}-\mathrm{Ag}$ heterometallic nanorods formed through a symmetrical growth of $\mathrm{Ag}$ on both ends of anisotropic Au nanorods (Fig. 1p, q). The segmented Ag-Au-Ag nanorods with an asymmetrical configuration can also be synthesized through kinetics control by tuning the reaction rate [60]. The segmented heterometallic nanostructures containing two plasmonic metals with a well-defined morphology and controlled symmetry may enable a variety of new applications [6163].

\section{METALLIC HETEROCRYSTALS WITH PLASMONIC AND CATALYTIC METALS}

The combination of plasmonic metals $(\mathrm{Au}, \mathrm{Ag}, \mathrm{Cu}, \mathrm{Al}$, etc.) with catalytic metals ( $\mathrm{Pt}, \mathrm{Pd}$, etc.) can utilize the advantages of efficient light-harvesting capability and local field enhancement from plasmonic metals as well as the highly active chemical surfaces from catalytic metals. Many interesting mechanisms have been proposed and 
exhibited in the hybrids of these two kinds of metals. For instance, the Nordlander and Halas group demonstrated a $\mathrm{Pd} / \mathrm{Al}$ heterometallic antenna-reactor complex that largely improved the light absorption and hot-carrier generation inside the Pd nanocrystals for photocatalysis $[11,64,65]$. Therefore, the controlled growth of metallic heterocrystals with plasmonic and catalytic metals is significant for many solar energy applications and has attracted intense attention.

Based on plasmonic metal nanocrystals with the desired size, shape, and SPR, many catalytic metals have been successfully overgrown on the plasmonic metal nanocrystals with a controlled configuration [66-71]. Wang et al. [66] designed and synthesized a $\mathrm{Pd} / \mathrm{Au}$ hybrid nanorod with multiple Pd nanoparticles heteroepitaxially grown onto an Au nanorod, which were mainly located at the two ends of the nanorod. As shown in Fig. 2a, the Pd nanoparticles tightly attached to the Au nanorods could gain electrons from the plasmon resonances of the $\mathrm{Au}$ nanorods. A Pd-catalyzed Suzuki coupling reaction is accelerated through plasmonic photocatalysis and photothermal heating. As a result, under the illumination of an $809 \mathrm{~nm}$ laser at $1.68 \mathrm{~W}$, the yield of the Suzuki coupling reaction was $\sim 2$ times that of the reaction performed at the same temperature with thermal heating. Some other heterocrystals with a similar configuration and plasmon-enhanced photocatalytic applications are also reported [72-98]. By altering the side-coating surfactants for the growth of Au nanorods, Zheng et al. [72] synthesized Pt-modified $\mathrm{Au}$ nanorods with the Pt covering the whole or only the ends of the nanorods (Fig. 2b, c). The anisotropic overgrowth of Pt for the Pttipped Au nanorods was achieved through building a close packing of surfactants at the sides of the nanorod by using a binary surfactant mixture consisting of cetyltrimethylammonium bromide (CTAB) and 5-bromosalicylic acid. The plasmon-enhanced hydrogen generation under visible to NIR illumination exhibited that the apparent quantum efficiency (AQE) of the Pt-tipped $\mathrm{Au}$ nanorods reached $0.51 \%$ at $540 \mathrm{~nm}$ and $0.68 \%$ at $940 \mathrm{~nm}$; the above results were due to the transfer of hot electrons from Au to Pt. Meanwhile, Pd-tipped and Pd-covered Au nanorods were also prepared for a plasmon-enhanced catalytic formic acid dehydrogenation at low temperatures [73]. Lou et al. [91] also prepared Pt-covered, Ptedged, and Pt-tipped Au triangular nanoprisms by controlling the overgrowth of Pt over the whole surface, and on the edges and the corners, respectively (Fig. 2d). An anisotropic growth of $\mathrm{Pt}$ was achieved with the help of selective adsorption of $\mathrm{I}^{-}$ions on $\mathrm{Au}(111)$ facets and the binding of $\mathrm{Ag}^{+}$to form $\mathrm{AgI}$ on the surface of $\mathrm{Au}$ [92]. The photocatalytic $\mathrm{H}_{2}$ generation under visible-NIR light irradiation shows that the $\mathrm{H}_{2}$ generation rate of the Ptedged $\mathrm{Au}$ triangular nanoprisms was 3 and 5 times higher than those of Pt-tipped and Pt-covered $\mathrm{Au}$ triangular nanoprisms due to a strong electric field and an increased interface for hot-electron transfer.

The deposition of catalytic metals on plasmonic metals can be precisely controlled in specific dimensions to form core-shell heterocrystals [12,99-104]. Aslam et al. [12] prepared a hybrid Pt@Ag core-shell nanocube with approximately six atomic layers of $\mathrm{Pt}$ shell, which corresponded to a thickness of $1.2 \pm 0.2 \mathrm{~nm}$ (Fig. 2e). The ultrathin Pt shells protected the unstable Ag core inside and provided chemically active surfaces. The measured and calculated optical extinction, absorption, and scattering of Ag and Pt@Ag nanocubes reveal that the energy of visible-light photons harvested by core plasmonic metals can selectively be flowed into the catalytically active centers on the nanostructure shell. The utility of these nanostructures for photocatalytic chemical reactions in a preferential oxidation of $\mathrm{CO}$ in excess $\mathrm{H}_{2}$ is demonstrated. In addition to the deposition of catalytic metals on plasmonic metals, the reverse configuration has also been developed [105-106]. Yuan et al. [105] reported the preparation of ultrathin PdAu alloy nanoshells with a controllable alloying degree on Pd nanocubes by a galvanic replacement reaction of Pd nanocubes mixed with $\mathrm{HAuCl}_{4}$, and a subsequent co-reduction of the dissolved $\mathrm{Pd}^{2+}$ and $\mathrm{AuCl}^{4-}$ (Fig. 2f, g).

Additionally, the configurations of heterocrystals combining plasmonic and catalytic metals have also been well controlled and exhibited in many cases with welldefined morphologies. Xu et al. [107] reported the growth of segmented $\mathrm{Pd}-\mathrm{Au}-\mathrm{Pd}$ nanorods with controllable sizes by growing $\mathrm{Pd}$ on both sides of $\mathrm{Au} \mathrm{dec-}$ ahedrons along the five-fold axial direction using $\mathrm{CTAB}$ and $\mathrm{I}^{-}$as growth modifiers (Fig. 2h). These Pd-Au-Pd nanorods showed a tunable SPR similar to that of the segmented Ag-Au-Ag nanorods [59-63,108,109]. Interestingly, $\mathrm{Pd}-\mathrm{Ag}$ [110] and $\mathrm{Pd}-\mathrm{Cu}$ [111] heterorods are synthesized through growing plasmonic metals of $\mathrm{Ag}$ or $\mathrm{Cu}$ onto only one side of Pd decahedrons (Fig. 2i). Xia's group also reported a kinetically controlled overgrowth of $\mathrm{Ag}$ [112,113], $\mathrm{Au}$ [114], and $\mathrm{Cu}$ [115] on Pd nanocrystal seeds to form hybrid dimers as well as nonconcentric and concentric bimetallic nanocrystals (Fig. 2j); the newly formed metallic atoms could be selectively nucleated and then epitaxially grown on a specific number of the six faces on a cubic Pd seed. 

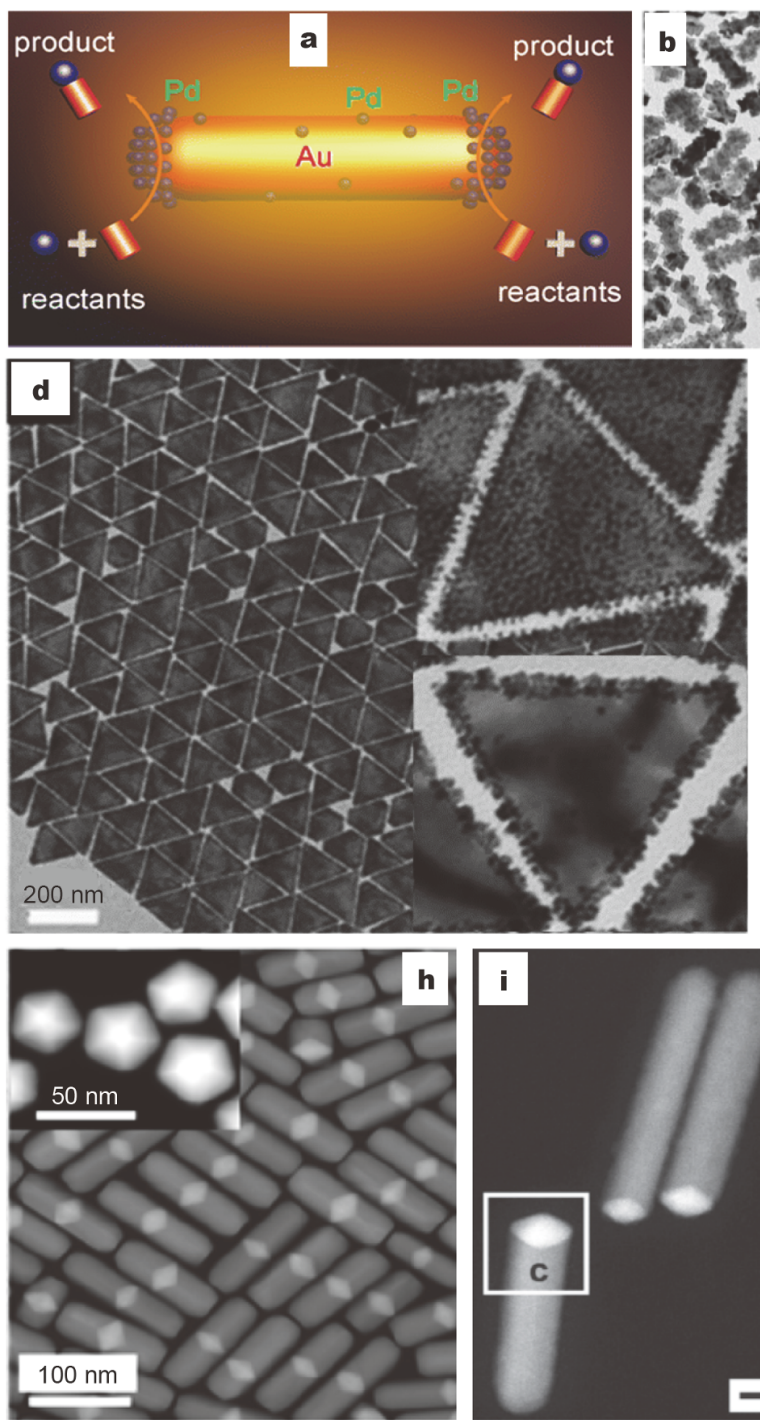
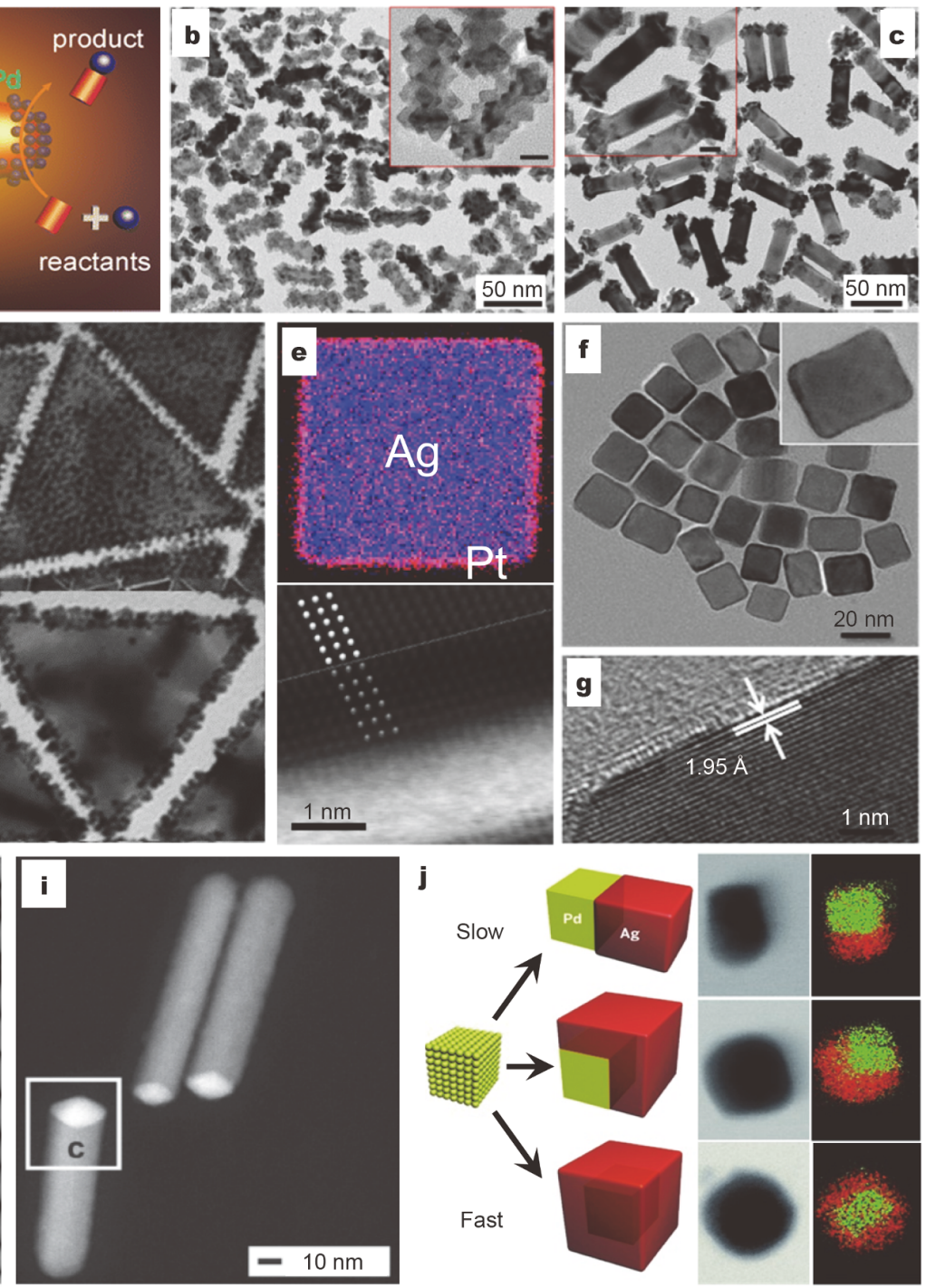

Figure 2 Heterostructures of plasmonic and catalytic metals. (a) Pd-modified Au nanorods for Suzuki coupling reactions by Wang et al. Reprinted with permission from Ref. [66]. Copyright 2013, American Chemical Society. Pt-covered (b) and Pt-tipped (c) Au nanorods for plasmon-enhanced hydrogen generation by Zheng et al. Reprinted with permission from Ref. [72]. Copyright 2014, American Chemical Society. (d) Pt-covered, Pt-edged, ant Pt-tipped Au nanotriangles with enhanced hot-electron transfer for $\mathrm{H}_{2}$ generation by Lou et al. Reprinted with permission from Ref. [91]. Copyright 2016, American Chemical Society. (e) Pt@Ag heterocubes with a controlled energy flow for photocatalytic CO oxidation by Aslam et al. Reprinted with permission from Ref. [12]. Copyright 2017, Springer Nature. (f, g) Ultrathin PdAu alloy nanoshells on $\mathrm{Pd}_{\text {nanocubes for } \mathrm{CO}_{2}}$ reduction by Yuan et al. Reprinted with permission from Ref. [105]. Copyright 2019, American Chemical Society. (h) Symmetrical Pd-Au-Pd segmented nanorods prepared by growing Pd on Au decahedrons by Xu et al. Reprinted with permission from Ref. [107]. Copyright 2016, American Chemical Society. (i) Asymmetrical Pd-Cu nanorods by Luo et al. Reprinted with permission from Ref.[111]. Copyright 2016, John Wiley \& Sons. (j) Site-selective growth of Ag on Pd nanocubes by controlling the reaction kinetics by Zeng et al. Reprinted with permission from Ref. [112]. Copyright 2012, John Wiley \& Sons.

\section{METAL-SEMICONDUCTOR HETEROSTRUCTURES WITH TWO COMPONENTS}

\section{Symmetric Morphology}

Overgrowth of semiconductor nanocrystals on plasmonic metals to obtain semiconductor-metal heterocrystals is much more difficult than that of metal-metal heterostructures. The remarkable achievement in this field is the overgrowth of monocrystalline CdS and CdSe semiconductor shells on Au nanoparticles [116,13]; these heterocrystals demonstrate a largely enhanced optical 
Stark effect and revised saturable absorption owing to the resonant interactions between plasmons and excitons [13]. Plasmon-plasmon coupling between a metal ( $\mathrm{Au})$ and a self-doped semiconductor $\left(\mathrm{Cu}_{2-x} \mathrm{~S}\right)$ was also observed in $\mathrm{Cu}_{2-x} \mathrm{~S} @ \mathrm{Au}$ heterorods [17]. In recent years, oxide semiconductors $\left(\mathrm{CeO}_{2}\right.$ and $\left.\mathrm{Cu}_{2} \mathrm{O}\right)$ and $2 \mathrm{D}$ semiconductors $\left(\mathrm{MoS}_{2}\right.$ and $\left.\mathrm{ReS}_{2}\right)$ have also been successfully overgrown on plasmonic metals, and enhanced photocatalytic activities have been reported [117-121]. Furthermore, controlling the morphology of the overgrown semiconductors on the metals has critical significance for second-order nonlinear responses and the photocatalysis efficiency.

Sun et al. [122] developed a general method for the preparation of sulfide shells on $\mathrm{Au}$ core nanoparticles under hydrothermal conditions (Fig. 3a). In this method, metal thiobenzoates decompose and provide sulfur for $\mathrm{Ag}^{+}$or $\mathrm{Cu}^{2+}$ ions to form sulfides that act as an adhesive layer for the deposition of other metal sulfides that are generated hydrothermally from the corresponding thiobenzoates. The preparation of hybrid nanostructures (CdS@Au, ZnS@Au) with CdS or ZnS shells on Au nanosphere cores has also been demonstrated via a direct decomposition of cysteine complexes with $\mathrm{Cd}^{2+}$ or $\mathrm{Zn}^{2+}$ ions [123-125]. Wang et al. [126] obtained $\mathrm{Co}_{x} \mathrm{~S} @ \mathrm{Au}$ core-shell nanoparticles by firstly forming Co@Au and then a subsequent sulfidation of the Co shell in octadecylamine. By combining the sulfidation or selenization of silver with a cation exchange, Zhang et al. [13,116] reported the nonepitaxial growth of monocrystalline CdS and CdSe nanoshells with precise structural and compositional tailoring on Au core nanoparticles (Fig. 3b, c).
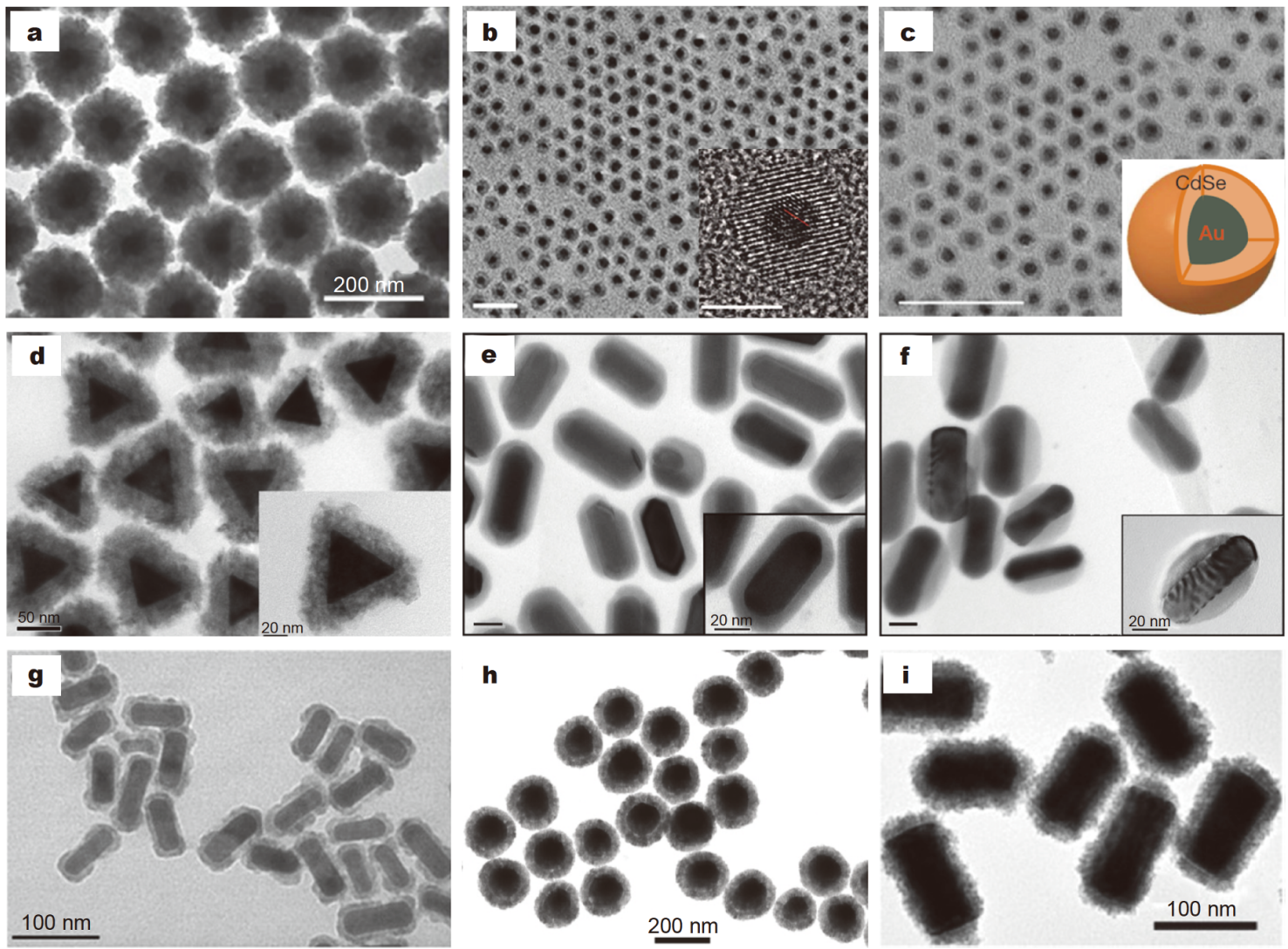

Figure 3 Overgrowing semiconductor shells on the metal cores. (a) Overgrowing ZnS nanocrystals on Au nanoparticles by Sun et al. Reprinted with permission from Ref. [122]. Copyright 2009, John Wiley \& Sons. Monocrystalline CdS (b) and CdSe (c) shells overgrown on Au nanoparticles (scale bar is $20 \mathrm{~nm}$ in (b) and $5 \mathrm{~nm}$ in the inset of (b), scale bar is $50 \mathrm{~nm}$ in (c)) by Zhang et al. Reprinted with permission from Ref. [116] and [13]. Copyright 2010, American Association for the Advancement of Science and Copyright 2010. Springer Nature, Respectively. (d) CdS nanocrystals on the Au nanotriplates by Ma et al. Reprinted with permission from Ref. [128], Copyright 2016, American Chemical Society. Closed (e) and end-opened (f) shells of $\mathrm{Ag}_{2} \mathrm{~S}$ on Au nanorods by Nan et al. Reprinted with permission from Ref. [129], Copyright 2014, John Wiley \& Sons. (g) Plasmonic semiconductor shells of $\mathrm{Cu}_{2-x} \mathrm{~S}$ on Au nanorods by Li et al. Reprinted with permission from Ref. [17]. Copyright 2018, John Wiley \& Sons. Oxide semiconductors of $\mathrm{Cu}_{2} \mathrm{O}(\mathrm{h})$ and $\mathrm{CeO}_{2}$ (i) on Au nanoparticles and nanorods by Zhang et al. Reprinted with permission from Ref. [131]. Copyright 2011, American Chemical Society, and Li et al. Reprinted with permission from Ref. [118]. Copyright 2014, American Chemical Society. 
Nonepitaxial growth is achieved by an overlayer of metal $\mathrm{Ag}$ shells, conversion to silver-compound shells $\left(\mathrm{Ag}_{2} \mathrm{X}\right)$ with an amorphous structure, and a cation exchange reaction to monocrystalline CdX. The above structures further demonstrate a plasmon-enhanced optical Stark effect induced by the resonant plasmon-exciton coupling in the core-shell nanostructures. Following these studies, sulfide shells are successfully grown on various Au nanostructures, such as triangles (Fig. 3d) and nanorods (Fig. 3e, f) [127-129]. Very recently, few-layer 2D semiconductors, such as $\mathrm{MoS}_{2}$ and $\mathrm{ReS}_{2}$, have also successfully been overgrown on $\mathrm{Au}$ nanoparticles, and very efficient charge transfer has been observed in this type of coreshell nanoparticle [119-121].

The growth of sulfide semiconductor shells can efficiently manipulate the nonlinear optical responses of $\mathrm{Au}$ nanocrystals. The complete, corner-opened, and endopened shells of $\mathrm{Ag}_{2} \mathrm{~S}$ on Au-core nanorods are obtained by controlling the position of an intermediate layer of $\mathrm{Ag}$ on the $\mathrm{Au}$ nanorods (Fig. 3e, f) [129]. The nonlinear absorption (NLA) is suppressed, and the nonlinear refraction (NLR) is largely enhanced after the overgrowth of the semiconductor shells. $\mathrm{Ag}_{2} \mathrm{~S} @ \mathrm{Au}$ heterorods with end-opened shells have the smallest saturation absorption with an NLA coefficient $\beta=-1.93 \mathrm{~cm} \mathrm{GW}^{-1}$ at the longitudinal SPR, which is only $35 \%$ that of the bare $\mathrm{Au}$ nanorods. $\mathrm{Ag}_{2} \mathrm{~S} @ \mathrm{Au}$ heterorods with complete shells demonstrate the largest NLR index $\gamma=-38.9 \times$ $10^{-4} \mathrm{~cm}^{2} \mathrm{GW}^{-1}$ at the longitudinal SPR, which is enhanced 3.1 times that of the bare Au nanorods. $\mathrm{Ag}_{2} \mathrm{~S} @ \mathrm{Au}$ heterorods with corner-opened shells display the largest NLR index $\gamma=-10.6 \times 10^{-4} \mathrm{~cm}^{2} \mathrm{GW}^{-1}$ at the transverse SPR, which is 8.6 times that of the bare Au nanorods. Interestingly, the overgrowth of complete CdS shells on $\mathrm{Au}$ nanorods leads to a sign reversal of the NLA coefficient $\beta$, varying it from -7.7 to $22.2 \mathrm{~cm} \mathrm{GW}^{-1}$ at the longitudinal SPR, while the NLR coefficient $\gamma$ increases from $-9.3 \times 10^{-4}$ to $-13.3 \times 10^{-4} \mathrm{~cm}^{2} \mathrm{GW}^{-1}$; the above results indicate a strong local field enhancement and plasmon-exciton interaction in the CdS@Au core-shell nanorods [15].

In particular, self-doped semiconductors, such as $\mathrm{Cu}_{2-x} \mathrm{~S}$ and $\mathrm{Cu}_{2-x} \mathrm{Se}$, demonstrate plasmon resonances in the NIR region, which can be used for studies of plasmon-plasmon coupling and photothermal effects $[17,127,130]$. Ji et al. [127] synthesized $\mathrm{Cu}_{2-x} \mathrm{~S} @ A u$ coreshell nanorods through a cation exchange reaction by substituting $\mathrm{Cd}^{2+}$ with $\mathrm{Cu}^{+}$based on nonepitaxially grown monocrystalline CdS on Au nanorods [116]. A plasmonenhanced photothermal effect of the $\mathrm{Cu}_{2-x} \mathrm{~S} @ \mathrm{Au}$ core- shell nanorods was demonstrated and harnessed for diverse HeLa cancer cell ablation applications in the NIR window. Li et al. [17] synthesized $\mathrm{Cu}_{2-x} \mathrm{~S} @ A u$ heterorods using a hydrothermal method and demonstrated a coupling effect between the two different kinds of plasmon resonances originating from the metal $\mathrm{Au}$ core and the $\mathrm{Cu}_{2-x} \mathrm{~S}$ semiconductor shell (Fig. $3 \mathrm{~g}$ ). By tuning the rod length and shell thickness, the coupled strength between the SPR of the $\mathrm{Cu}_{2-x} \mathrm{~S}$ shells and the longitudinal SPR of the Au nanorod cores was measured to be $180 \mathrm{meV}$. This plasmon coupling effect disappears for the prepared $\mathrm{Cu}_{2} \mathrm{~S} @ \mathrm{Au}$ heterorods without self-doping $(x=0)$.

Heterostructures with oxide semiconductor shells on plasmonic metal cores also exhibit unique properties and potential applications ranging from photocatalysis to optical devices [117,118,131-134]. Various oxide semiconductor shells have been synthesized, such as $\mathrm{TiO}_{2}$, $\mathrm{Cu}_{2} \mathrm{O}, \mathrm{Fe}_{2} \mathrm{O}_{3}, \mathrm{ZnO}$, and $\mathrm{CeO}_{2}$. Here, we only introduce core-shell $\mathrm{Cu}_{2} \mathrm{O} @ \mathrm{Au}$ nanoparticles and $\mathrm{CeO}_{2} @ \mathrm{Au}$ nanorods as two examples. $\mathrm{Cu}_{2} \mathrm{O}$ is a promising p-type semiconductor for solar energy harvesting applications. Zhang et al. [131] synthesized $\mathrm{Cu}_{2} \mathrm{O} @ \mathrm{Au}$ core-shell nanoparticles with geometrically tunable optical properties by the hierarchical assembly of $\mathrm{Cu}_{2} \mathrm{O}$ nanocrystallites and the formation of a polycrystalline $\mathrm{Cu}_{2} \mathrm{O}$ nanoshell wrapped around the Au core (Fig. 3h). By controlling the geometrical parameters, such as the thickness of the $\mathrm{Cu}_{2} \mathrm{O}$ shell, the size of the Au core and the spacing between the core and shell, the light absorption and scattering can be tuned over a broad spectral range from the visible to NIR region. In addition, the transverse dipolar and octupolar SPRs appear as a result of the anisotropic morphology; furthermore, the coupling between the core and the high dielectric constant of the shell can be tuned by the gap between the $\mathrm{Au}$ core and $\mathrm{Cu}_{2} \mathrm{O}$ shell [132]. Metal- $\mathrm{Cu}_{2} \mathrm{O}$ core-shell nanoparticles also exhibit efficient photocatalytic activities compared with that of pristine metal or $\mathrm{Cu}_{2} \mathrm{O}$ nanoparticles because of a plasmon-induced resonant energy transfer and direct hot-electron transfer $[133,134]$. The oxide semiconductor $\mathrm{CeO}_{2}$ has been widely used as an ultraviolet absorber and an electrolyte for fuel cells, and $\mathrm{CeO}_{2}$ and $\mathrm{Au}$ can form an appropriate Schottky barrier to facilitate hot-electron injection. Li et al. [118] reported a general method to grow uniform ceria on Au nanocrystals through the heterogeneous nucleation and growth of $\mathrm{CeO}_{2}$ by forming $\mathrm{Ce}^{3+}$-EDTA (ethylenediaminetetraacetic acid, EDTA) complex ions with a slow hydrolysis and oxidation of $\mathrm{Ce}^{3+}$ (Fig. 3i). Under visible light, the $\mathrm{CeO}_{2} / \mathrm{Au}$ core-shell nanorods show good photocatalytic activities of selectively transforming benzyl 
alcohol to benzaldehyde. The Au core works as a plasmonic component for efficient light harvesting, which leads to both photothermal conversion and hot-electron injection, while the $\mathrm{CeO}_{2}$ shell can provide catalytically active sites for the oxidation reaction.

\section{Asymmetric Morphology}

Morphology control of the semiconductor nanocrystals that are overgrown on plasmonic nanostructures has critical significance for photocatalytic applications. For asymmetric metal-semiconductor heterocrystals with both components exposed to the outside, the reactive hotspots, metal plasmons and quantum-confined electronic states in the semiconductor nanostructures are tactfully integrated in one nanosystem [135-139]. Great efforts have been made to explore the physical and chemical properties observed with asymmetric heterostructures [140-146]. An abundance of synergistic properties from the asymmetric heterocrystals have received wide attention, such as the heterojunction-induced photogenerated carrier separation, the plasmon-enhanced sunlight harvesting and the plasmon-induced hot-electron transfer from metal nanostructures into strong coupled semiconductors [147-150]. The essence of asymmetric synthesis for metal-semiconductor heterostructures is selective growth of the shell, which comes from the anisotropic growth environments and kinetics parameters. A large number of schemes keep emerging toward the synthesis of asymmetric metal-semiconductor heterostructures, including those based on selective anisotropic growth [151-161], oxidation-directed decomposition [135], phase segregation induced by ripening $[162,163]$, an assembly welding process, photochemical fabrication [164], in situ sulfuration [154], and cationexchange technology $[155,116]$.

Zhao et al. [165] comparatively deposited CdS nanocrystals on Au nanoparticles with concentric core/shell, nonconcentric core/shell, and heterodimer morphologies. The various morphologies were achieved by tuning the crystallinity of $\mathrm{Ag}_{2} \mathrm{~S}$ in an intermediate step. An amorphous $\mathrm{Ag}_{2} \mathrm{~S}$ leads to a core/shell morphology, while a crystalline $\mathrm{Ag}_{2} \mathrm{~S}$ results in a nonconcentric core/shell morphology and then Au-CdS heterodimers. The hydrogen generation efficiency of semishelled CdS/Au heterodimers is much larger than that of CdS@Au core-shell heterostructures since both $\mathrm{Au}$ and $\mathrm{CdS}$ are involved in the charge transfers that generate hydrogen (Fig. 4a). Very recently, Liu et al. [19] synthesized $\mathrm{Cu}_{2-x} \mathrm{Se} / \mathrm{Au}$ heterodimer nanoparticles for deep tissue imaging by initiating nucleation and growth of a $\mathrm{Cu}_{2-x}$ Se nanocrystal domain on each $\mathrm{Au}$ seed through a gradually increasing reaction temperature (Fig. 4b). Liu et al. [166] obtained $\mathrm{CdSe} / \mathrm{Au}$ Janus nanoparticles with a high-quality interface using an etching effect during the overgrowth of $\mathrm{CdSe}$. The hydrogen generation efficiency of the CdSe/Au Janus nanoparticles increases by 3.9 times compared with that of the $\mathrm{CdSe} / \mathrm{Au}$ heteroparticles with semishells of $\mathrm{CdSe}$, which indicates that the interfaces strongly influence carrier transfer in the heterostructures. Liang et al. [167] reported the controlled overgrowth of CdSe nanocrystals on one side, one tip or two tips of Au nanorods (Fig. 4c-e). The morphology tuning was achieved by selectively overgrowing a mediated layer of $\mathrm{Ag}$ on $\mathrm{Au}$ nanorods and controlling the $\mathrm{pH}$ value of the reaction solution (deposition rate) for the overgrowth of CdSe. Wang et al. [168] achieved a tip and side growth of CdSe on Au nanorods by improving Liang's method and controlling the concentration of $\mathrm{Cd}^{2+}$. These two growth modes lead to strong interactions between excitons of CdSe and the transverse or longitudinal SPRs of Au nanorods. The local field of the CdSe nanocrystals overgrown on the one side of Au nanorods is enhanced by the transverse SPR in the visible region. The side-growth mode also leads to a larger interface between the $\mathrm{Au}$ and CdSe compared with that of the tip-growth mode; eventually, the hydrogen production efficiency of the sidegrown $\mathrm{CdSe} / \mathrm{Au}$ heterorods is 2.2 times that of the tipgrown $\mathrm{CdSe} / \mathrm{Au}$ heterorods as well as being 26.4 times that of the pure Au nanorods. These studies reveal the significance of morphology as well as the interface of the metal-semiconductor heterostructures on photocatalytic hydrogen generation.

Based on anisotropic metal nanocrystals (such as $\mathrm{Ag}$ and $\mathrm{Cu}$ ) with low chemical stability, asymmetric heterocrystals can be directly synthesized by selective sulfuration or oxidation without assistance of a mediation layer. Zeng et al. [154] reported $\mathrm{Ag}_{2} \mathrm{~S} / \mathrm{Ag}$ hybrid nanocrystals prepared by site-selective sulfuration at the corner sites of nanotriangles and nanotubes of Ag, which greatly improved their stability in air. Liu et al. [169] reported two types of $\mathrm{Cu}_{x} \mathrm{~S} / \mathrm{Cu}$ heterocrystals (heterodimers and hetero-oligomers) produced from $\mathrm{Cu}$ nanocrystals with alkanethiol as both a surfactant and a sulfur source; the above method utilized the high reactivity toward sulfuration of the $\mathrm{Cu}$ nanocrystals.

The anisotropic growth of oxides, such as $\mathrm{TiO}_{2}$ and $\mathrm{CeO}_{2}$, on $\mathrm{Au}$ nanorods have been reported. As shown in Fig. 4f, Wu et al. [170] obtained $\mathrm{TiO}_{2} / \mathrm{Au}$ nanodumbbells with spatially separated $\mathrm{Au}$ and $\mathrm{TiO}_{2}$ regions with the help of a high curvature and low density of a CTAB 


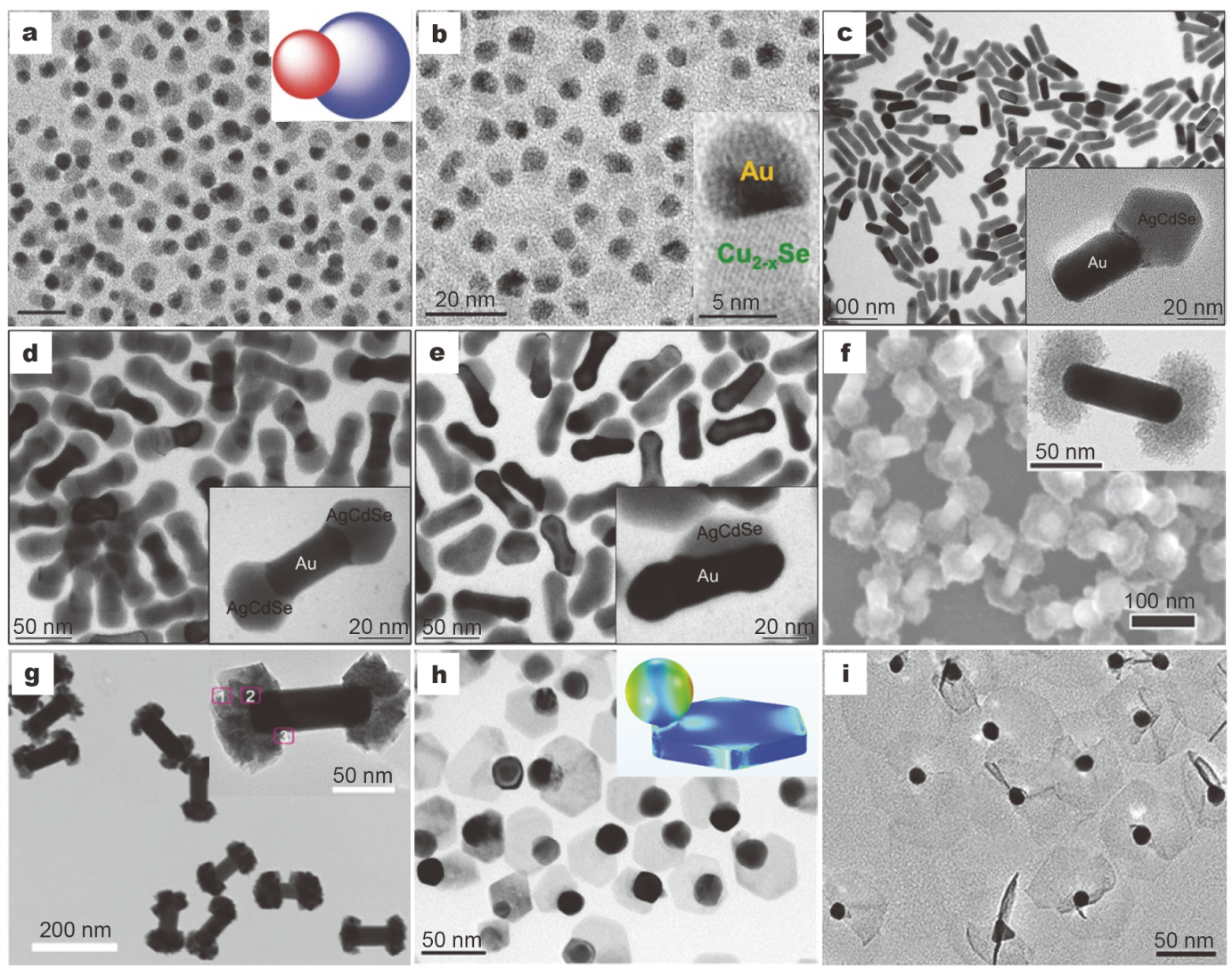

Figure 4 Morphology control of semiconductor nanocrystals overgrown on metal nanostructures. (a) CdS/Au heterostructures with CdS semishells coated on Au nanoparticles (scale bar, $20 \mathrm{~nm}$ ) by Zhao et al. Reprinted with permission from Ref. [165]. Copyright 2014, John Wiley \& Sons. (b) $\mathrm{Cu}_{2-x} \mathrm{Se} / \mathrm{Au}$ Janus nanoparticles with an etching effect on the interfaces by Liu et al. Reprinted with permission from Ref. [19]. Copyright 2013, American Chemical Society. Controlled growth of AgCdSe nanocrystals on one tip (c), two tips (d), and one side (e) of Au nanorods with an etching effect by Liang et al. Reprinted with permission from Ref. [167]. Copyright 2012, American Chemical Society. Oxide semiconductor nanocrystals of $\mathrm{TiO}_{2}$ (f) and $\mathrm{CeO}_{2}$ (g) overgrown around the two ends of Au nanorods with excellent photocatalytic efficiency by Wu et al. (Reprinted with permission from Ref. [170]. Copyright 2016, American Chemical Society), and by Jia et al. (Reprinted with permission from Ref. [171]. Copyright 2019, American Chemical Society). Ultrathin nanosheets of CuSe (h) and $\mathrm{MnO}_{2}$ (i) overgrown on Au nanoparticles by Ma et al. (Reprinted with permission from Ref. [172], Copyright 2019, John Wiley \& Sons), and by Liu et al. (Reprinted with permission from Ref. [173], Copyright 2016, Royal Society of Chemistry).

surfactant at the tips. Compared with the $\mathrm{TiO}_{2} @ \mathrm{Au}$ coreshell nanorods, the $\mathrm{TiO}_{2} / \mathrm{Au}$ nanodumbbells present significantly more efficient photocatalytic $\mathrm{H}_{2}$ evolution and dye degradation, which is attributed to a hot-electron transfer from the plasmonic metal to the semiconductor across a Schottky junction formed between the $\mathrm{TiO}_{2}$ and Au. Jia et al. [171] also reported a strategy to selectively coat crystalline $\mathrm{CeO}_{2}$ on $\mathrm{Au}$ nanorods (Fig. 4g). Due to the smaller steric hindrance at the ends than at the side surfaces, $\mathrm{K}_{2} \mathrm{PtCl}_{4}$ may preferentially adsorb at the ends of $\mathrm{Au}$ nanorods, triggering an autoredox reaction with the ceria precursor at the ends to form end $-\mathrm{CeO}_{2} / \mathrm{Au}$ heterorods. Compared with the $\mathrm{CeO}_{2} @ \mathrm{Au}$ core-shell nanostructures, these end- $\mathrm{CeO}_{2} / \mathrm{Au}$ heterorods offer reaction sites for both reduction and oxidation. Under plasmon excitation, the Au nanorods absorb NIR light to generate hot electrons and hot holes. The produced hot electrons are injected into the $\mathrm{CeO}_{2}$ to reduce $\mathrm{N}_{2}$ into $\mathrm{NH}_{3}$, meanwhile, the hot holes are consumed at the side surface of the Au nanorods. In contrast, in the core-shell nanostructures, the $\mathrm{Au}$ nanorods are buried inside and $\mathrm{CH}_{3} \mathrm{OH}$ has difficulty accessing the hot holes, thus resulting in a useless electron-hole recombination. The end$\mathrm{CeO}_{2} / \mathrm{Au}$ heterorods exhibit an excellent performance with a rate constant of $0.68 \times 10^{-2} \mathrm{~min}^{-1}$, which is an 8.9and 5.0-fold increase compared with that of the bare $\mathrm{Au}$ nanorods and the $\mathrm{CeO}_{2} @ \mathrm{Au}$ core-shell nanostructures, respectively; the above results suggest that the spatially separated structure facilitates electron-hole separation.

Overgrowing semiconductor nanosheets (2D mor- 
phology) on plasmonic metal has also attracted considerable attention owing to their unique photoelectronic properties. Ma et al. [172] reported the synthesis of $\mathrm{CuSe/}$ Au hybrids, where CuSe nanosheets were grown along the tangent of Au nanospheres by a convenient hydrothermal method (Fig. 4h). In detail, by adding Au nanospheres into a mixture solution of cupric nitrate, NaHSe and $\mathrm{CTAB}$, the CuSe nanosheets would grow on the surface of the Au nanospheres. Then, they would enlarge along the tangent of the $\mathrm{Au}$ nanospheres. Under light irradiation at 550 and $950 \mathrm{~nm}$, the CuSe/Au tangential hybrids showed stronger photocatalytic hydrogen generation than that of a mixture of $\mathrm{Au}$ and $\mathrm{CuSe}$. The strong enhancement originates from the dual-plasmon coupling as well as the synergistic effect between the plasmon and exciton. Liu et al. [173] successfully prepared the $\mathrm{MnO}_{2} / \mathrm{Au}$ nanosheets by a hydrothermal reduction of $\mathrm{KMnO}_{4}$ on $\mathrm{Au}$ nanoparticles (Fig. 4i). During the growth process of $\mathrm{MnO}_{2} / \mathrm{Au}$ nanosheets, citrate-capped AuNPs act as a nucleation center and the trace sodium citrate on the AuNP surface performs as a reducing agent for $\mathrm{KMnO}_{4}$. The $\mathrm{MnO}_{2} / \mathrm{Au}$ nanosheets exhibit largely enhanced electrocatalytic activity of 30-40 times compared with that of pure $\mathrm{MnO}_{2}$ nanosheets and $\mathrm{Au}$ nanoparticles due to the strong interaction between the $\mathrm{Au}$ nanoparticles and $\mathrm{MnO}_{2}$ nanosheets.

\section{METAL-SEMICONDUCTOR HETEROSTRUCTURES WITH THREE COMPONENTS}

Semiconductor-metal heterostructures with multiple components integrate more functionalities and have better performance than those with two components [174-193]. The plasmonic and catalytic metals combined with a semiconductor, such as (CdS-Pt)@Au heterotriangles and $\mathrm{Au} / \mathrm{XS}_{2} @ \mathrm{Au}(\mathrm{X}=\mathrm{Mo}, \mathrm{Re})$ heteroparticles, demonstrate considerably improved photocatalytic activities $[22,120]$. On the other hand, two semiconductors with vertical or lateral configurations on a plasmonic metal have also been presented and exhibit configurationdependent functions and applications. For vertical heterocrystals, such as $\mathrm{CdS}_{-} \mathrm{AgS}_{2}-\mathrm{Ag}$ heterorods and PbS@AgS @Au core-multishell nanorods, the middle material acts as a spacer, while the outer material is a functional semiconductor; the spacer enhances the functional material, which then provides a better performance on fluorescence, nonlinear responses, and photocatalytic activities [194,195]. For lateral heterocrystals, such as $\left(\mathrm{Cu}_{2-x} \mathrm{~S}-\mathrm{CdS}\right) @ \mathrm{Au}$, two semiconductors construct a p-n junction shell, and both semiconductors are directly overgrown on the Au core, resulting in a significantly improved photocatalytic efficiency [23].

For the instance of photocatalysis, Chen et al. [120] synthesized Au/XS $@$ @Au heterostructures (Fig. 5a), which contain a plasmonic antenna (large Au-core nanoparticles), a semiconductor shell (2D XS $)$, and a catalytic reactor (small $\mathrm{Au}$ nanoparticles), through coating fewlayer $\mathrm{XS}_{2}\left(\mathrm{MoS}_{2}\right.$ and $\left.\mathrm{ReS}_{2}\right)$ on 40-nm Au nanoparticles by a hydrothermal method and depositing small gold nanoparticles on the $\mathrm{XS}_{2}$ layers via a photoreduction of $\mathrm{HAuCl}_{4}$. The ultrathin $2 \mathrm{D} \mathrm{XS}$ layers work as a spacer between the antenna and reactor, which also increases the charge transfer and carrier separation in the reactors and notably enhances the photocatalytic activity of the heterostructures. With visible light irradiation, the $\mathrm{Au} /$ $\mathrm{ReS}_{2} @ \mathrm{Au}$ and $\mathrm{Au} / \mathrm{MoS}_{2} @ \mathrm{Au}$ heterostructures exhibit enhanced photocatalytic hydrogen generation rates of 3.59- and 2.66-fold compared with that of the sum of the three individual components, respectively. Ma et al. [22] synthesized (CdS-Pt)@Au heterotriangles, which also contain a plasmonic antenna (Au triangles), a catalytic reactor ( $\mathrm{Pt}$ around three corners of the Au triangles), and a semiconductor (CdS on the surface of $\mathrm{Au}$ triangles). The (CdS-Pt)@Au heterotriangle was achieved by selectively growing $\mathrm{Pt}$ nanoparticles onto the three tips that were covered by fewer CTAB molecules on the Au nanotriangles and then depositing a CdS shell on the remaining sites. The three components ( $\mathrm{Au}, \mathrm{Pt}$, and $\mathrm{CdS}$ ) are in contact with each other, providing multiple channels of charge transfer (Fig. 5b). Under visible light irradiation, the (CdS-Pt)@Au heterotriangles exhibit significantly improved photocatalytic hydrogen generation efficiency, which is approximately 2.5 and 1.4 times higher than those of Pt/CdS and Pt/CdS@Au, respectively.

Nonlinear optical responses can be enhanced in finely designed multiple-component heterocrystals. In coremultishell heterostructures of $\mathrm{PbS} @ \mathrm{Ag}_{2} \mathrm{~S} @ \mathrm{AuAg} @ \mathrm{Au}$ (shown in Fig. 5c) [195], the middle spacer layer $\left(\mathrm{Ag}_{2} \mathrm{~S}\right)$ has a relatively low refractive index, and the out-layer shell $(\mathrm{PbS})$ has a very high refractive index. The coremultishell heterostructures were synthesized by a rationally designed multiple-step method involving the deposition of Ag shells onto the surfaces of the Au nanorod seeds; the above structure was achieved through the formation of $\mathrm{AuAg}$ shells by a galvanic replacement reaction, and then the overgrowth of $\mathrm{Ag}_{2} \mathrm{~S}$ and $\mathrm{PbS}$ shells. The $\mathrm{Ag}_{2} \mathrm{~S}$ shell and the air gap inside serve as double light-trapping layers that efficiently confine and enhance the local field within the colloidal nanostructures. Open- 


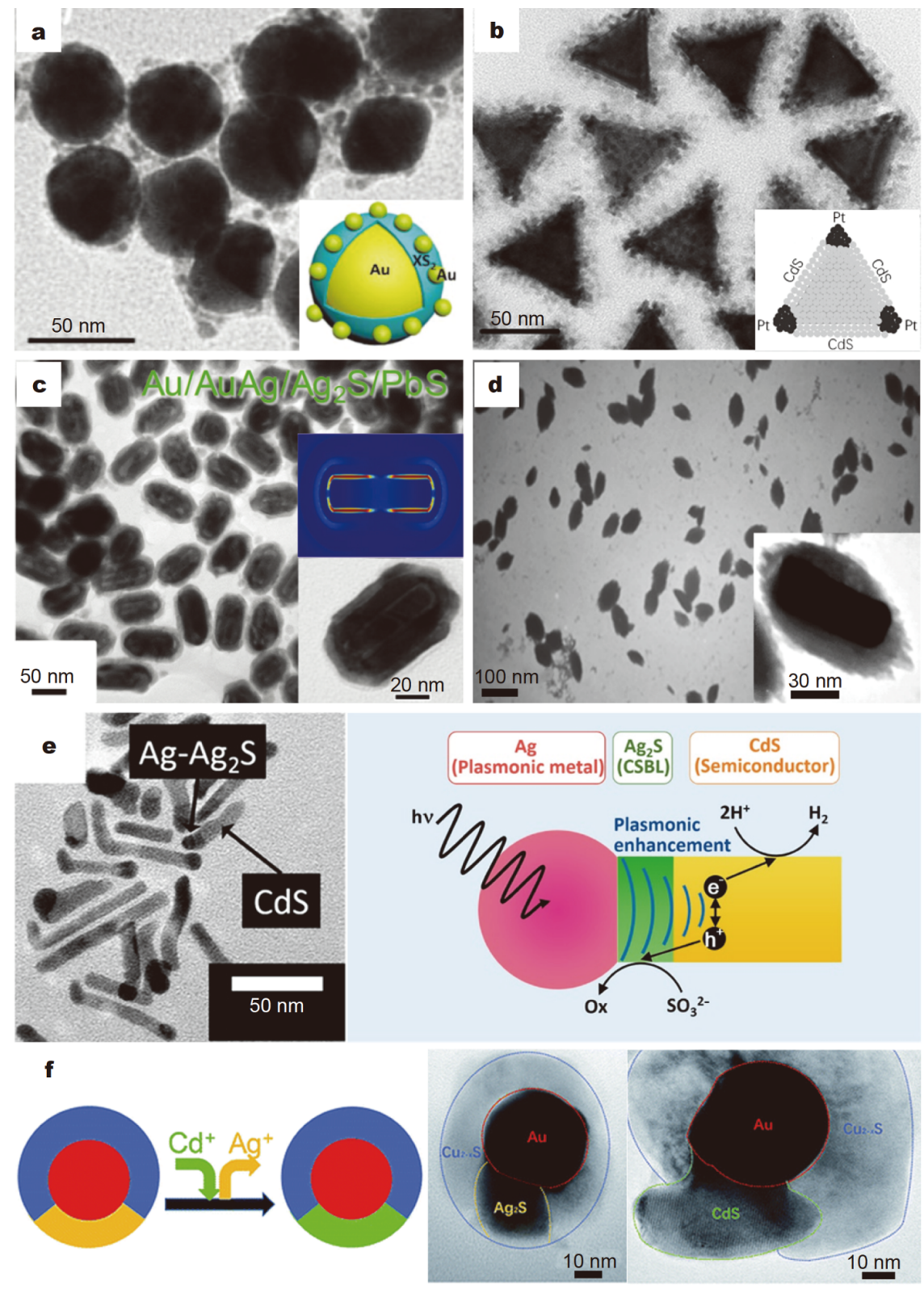

Figure 5 Metal-semiconductor heterostructures with three components. (a) $\mathrm{Au} / \mathrm{XS}_{2} @ \mathrm{Au}(\mathrm{X}=\mathrm{Mo}, \mathrm{W}, \mathrm{Re})$ heterostructures with an antenna and reactor separated by an ultrathin $2 \mathrm{D} \mathrm{XS}_{2}$ layer by Chen et al. Reprinted with permission from Ref. [120]. Copyright 2018, The Royal Society of Chemistry. (b) (CdS-Pt)@Au heterostructures with multichannel charge transfer by Ma et al. Reprinted with permission from Ref. [22]. Copyright

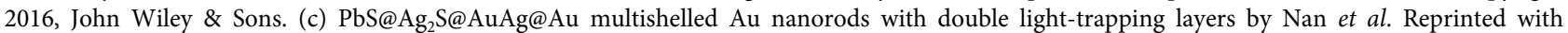
permission from Ref. [195]. Copyright 2016, The Royal Society of Chemistry. (d) CdS quantum dots on $\mathrm{Ag}_{2} \mathrm{~S} @ \mathrm{Au}$ core-shell nanorods with $\mathrm{Ag}_{2} \mathrm{~S}$ as a spacer layer for fluorescence enhancement by Sancholi et al. Reprinted with permission from Ref. [196]. Copyright 2019, Elsevier. (e) CdS nanorods on $\mathrm{Ag}$ nanoparticles with $\mathrm{Ag}_{2} \mathrm{~S}$ as a carrier-selective blocking layer for enhancement of the hydrogen evolution reaction by Kawawaki et al. Reprinted with permission from Ref. [194]. Copyright 2019, American Chemical Society. (f) $\mathrm{Cu}_{2-x} \mathrm{~S}$-CdS p-n junction on Au nanoparticles with plasmonenhanced photocatalytic activities by Ma et al. Reprinted with permission from Ref. [23]. Copyright 2019, The Royal Society of Chemistry.

aperture Z-scan and time-resolved pump-probe measurements both demonstrate suppressed saturable absorption in the PbS@Ag $\mathrm{S} @ \mathrm{AuAg} @ \mathrm{Au}$ core-multishell heterostructures. The nonlinear refraction index $\gamma$ of the bare Au nanorods is $-0.12 \times 10^{-4} \mathrm{~cm}^{2} \mathrm{GW}^{-1}$ and that of $\mathrm{PbS} @ \mathrm{Ag}_{2} \mathrm{~S} @ \mathrm{AuAg} @ \mathrm{Au}$ is $-1.14 \times 10^{-4} \mathrm{~cm}^{2} \mathrm{GW}^{-1}$, which shows an enhancement of 9.5 times. The large enhancement of $\gamma$ is mainly attributed to the strong field con- 
finement in the core-multishell heterostructures. Correspondingly, the one-photon figure of merit (FOM) of PbS@Ag $\mathrm{S} @ A u A g @ A u$ heterostructures is enhanced by 5 times, while the two-photon FOM is decreased by $80 \%$. The above results indicate that the one- and two-photon FOMs are significantly improved and suitable for applications in all-optical switching. Additionally, the influence of the surrounding environment is very small since the field enhancement around the interface of the outer layer and the surrounding medium is very weak.

In many three-component heterocrystals, the interlayer between the metal and semiconductor plays a critical role in delivering a good performance in many applications. The middle shell $\mathrm{Ag}_{2} \mathrm{~S}$ in the CdS@ $\mathrm{Ag}_{2} \mathrm{~S} @ A u$ heterorods acts as a spacer between the CdS quantum dots and $\mathrm{Au}$ nanorods (Fig. 5d) [196], which efficiently suppresses the fluorescence quenching effect. The corresponding fluorescence peak of the CdS quantum dots is tuned from 530 to $580 \mathrm{~nm}$, which is much stronger than the NIR fluorescence of $\mathrm{Ag}_{2} \mathrm{~S}$, which is in a range of $750-950 \mathrm{~nm}$. The semiconductor $\mathrm{Ag}_{2} \mathrm{~S}$ can also work as a carrier-selective blocking layer between Ag nanoparticles and CdS nanorods in the heterostructures of CdS- $\mathrm{Ag}_{2} \mathrm{~S}-\mathrm{Ag}$ (Fig. 5e) [194]. CdS- $\mathrm{Ag}_{2} \mathrm{~S}-\mathrm{Ag}$ was prepared by a seeded growth of $\mathrm{CdS}$ on $\mathrm{Ag}_{2} \mathrm{~S}$ seed nanoparticles and a subsequent chemical extraction of $\mathrm{S}^{2-}$ ions from $\mathrm{Ag}_{2} \mathrm{~S}$. The $\mathrm{Ag}_{2} \mathrm{~S}$ layer synergistically improves the plasmonic enhancement effect of $\mathrm{Ag}$ on the hydrogen evolution reaction of CdS by both a selective blocking of photoexcited electrons and an effective transfer of holes. The above mechanism extends the lifetime of the electrons in the conduction band in the CdS semiconductor photocatalyst and leads to a 33-fold enhancement of the hydrogen evolution reaction.

Moreover, two semiconductors constructing a vertical bilayer or a lateral $\mathrm{p}-\mathrm{n}$ junction on a plasmonic metal may prominently improve their photocatalytic efficiency. For instance, Ma et al. [197] synthesized CdS@Bi $\mathrm{S}_{3} @ \mathrm{Au}$ dumbbell-like heterostructures by successively growing $\mathrm{Bi}_{2} \mathrm{~S}_{3}$ shells and CdS shells onto $\mathrm{Au}$ cores through a possible chelation of $\mathrm{CTAB}$ and ascorbic acid molecules with metal ions to overcome a lattice mismatch. Dumbbell-like $\mathrm{Au}$ was formed due to a rate difference in $\mathrm{Au}$ overgrowth (higher overgrowth rate along the $\{111\}$ direction) on the different facets of CTAB-stabilized Au nanorods. The dumbbell-like Au cores prominently increase the absorption in the visible region via transverse SPR, and the energy alignment of $\mathrm{CdS} @ \mathrm{Bi}_{2} \mathrm{~S}_{3}$ has the advantage for carrier separation in photocatalytic processes. Very recently, Ma et al. [23] developed a three-step method to grow $\mathrm{CdS}-\mathrm{Cu}_{2-x} \mathrm{~S}$ lateral heteroshells on $\mathrm{Au}$ nanoparticles (Fig. 5f). The synthesis strategy for lateral heteroshells was realized step-by-step through the growth of incomplete $\mathrm{Ag}_{2} \mathrm{~S}$ shells, followed by $\mathrm{Cu}_{2-x} \mathrm{~S}$ growth to complete the shells, and then a selective cation exchange process from $\mathrm{Ag}_{2} \mathrm{~S}$ to CdS. All the components in this complex nanostructure are directly connected with each other. CdS is an n-type semiconductor with a bandgap of $2.4 \mathrm{eV}$ and good photocatalytic activity, while $\mathrm{Cu}_{2-x} \mathrm{~S}$ is a p-type semiconductor with a stoichiometry-dependent bandgap. $\mathrm{CdS}-\mathrm{Cu}_{2-x} \mathrm{~S}$ has a type II band alignment as well as $\mathrm{p}-\mathrm{n}$ junction properties, so the electrons can transfer to the conduction band of $\mathrm{CdS}$ and the holes transfer to the valence band of $\mathrm{Cu}_{2-x} \mathrm{~S}$ in the photocatalytic process. The $\mathrm{Au}$ cores in this nanostructure have plasmon-mediated effects such as light harvesting in the visible region, a plasmon-enhanced local field, hot-electron generation, and energy transfer from a metal to a semiconductor. Owing to the two plasmonic materials ( $\mathrm{Au}$ and $\mathrm{Cu}_{2-x} \mathrm{~S}$ ), the structure can absorb the light in the visible region and NIR region efficiently, and the $p-n$ junction leads to good separation of the photon-excited electron-hole pair. The (CdS- $\left.\mathrm{Cu}_{2-x} \mathrm{~S}\right) @ A u$ heteroparticles show a high conversion efficiency of $22.4 \%$ and a high apparent quantum efficiency in photocatalytic dye degradation of $1.68 \times$ $10^{-12} \mathrm{~mol} \mathrm{~mW}^{-1} \mathrm{~h}^{-1}$.

\section{SUMMARY AND OUTLOOK}

In this review, we focus on the controlled growth and applications of plasmonic-metal-involved heteronanostructures. Many bicomponent hybrids, including plasmonic metal/plasmonic metal, plasmonic metal/catalytic metal, plasmonic metal/semiconductor, and multiple-component hybrids, are introduced according to their synthesis methods, characteristic properties and excellent performances in applications. The interaction between the different materials allows the plasmonic properties to be extended and enhanced by tuning the plasmonic wavelength and intensity as well as introducing a new plasmonic mode. The unique properties of plasmonic materials, including local field enhancement, intense optical absorption and scattering, and hot-electron generation and transfer, can dramatically enhance the properties of the adjacent materials and improve the performances of the hybrids. It should be noted that the properties and performances of the hybrids are highly dependent on the configuration, morphology, and dimension. Therefore, a controlled growth of plasmonic heterostructures is significantly important for their applications in a large variety of fields.

Controlled growth of colloidal metal nanostructures to 
satisfy the optimized design of plasmonic antennas is still a challenge, since the theoretically optimized antennas have a 3D hollow nanostructure consisting of an open cavity that supports magnetic plasmon resonance for farfield coupling and a hotspot for near-field coupling [198]. Colloidal heterocrystals consisting of two plasmonic metals offer a possible approach to satisfy these theoretical demands [199]; the above configuration can also support multifrequency plasmon resonances for the enhancement of both linear and nonlinear optical processes, especially the enhancement of second-harmonic generation (SHG) by combining electron and magnetic plasmon resonances.

The combination of plasmonic metal and catalytic metal can make use of a large local field enhancement in the plasmonic metal to improve the light absorption of the catalytic metal through a "forced plasmon" effect in a non-contact condition; the above combination can also take advantage of plasmon-induced hot-electron generation and transfer to enrich the active electrons in the catalytic metal for chemical reactions on its surface in an intimate-contact condition. The atomic-scale arrangement, especially on surfaces with tunable electronic structures and electron transfer, is also important for understanding the chemical reaction processes in catalytic applications. Site-selective growth with a precisely controlled size and stoichiometric ratio is highly expected for photocatalysis and solar-to-chemical energy transfer.

For metal-semiconductor heterostructures, controlling the morphologies of both the metal and semiconductor as well as their interface directly influence their plasmonic and catalytic performances. Further studies may focus on the following three aspects. i) Enhancing coupling strengths of plasmon-exciton, exciton-plasmon-exciton, and plasmon-plasmon of metals and doped semiconductors in heterostructures by tuning the resonances of plasmon(s) and exciton(s). ii) Enhancing excitonic fluorescence by optimizing radiative and nonradiative processes in colloidal heterostructures used in multifunctional biolabeling. iii) Enhancing excitation energy and charge transfer in heterocatalysts, especially by overgrowing ultrathin $2 \mathrm{D}$ nanomaterials at a low temperature, searching for an appropriate carrier-selective blocking layer, and overgrowing lateral $\mathrm{p}-\mathrm{n}$ junctions on a plasmonic metal.

Received 9 December 2019; accepted 28 January 2020; published online 5 March 2020

1 Kelly KL, Coronado E, Zhao LL, et al. The optical properties of metal nanoparticles: the influence of size, shape, and dielectric environment. J Phys Chem B, 2003, 107: 668-677

2 Xia Y, Xiong Y, Lim B, et al. Shape-controlled synthesis of metal nanocrystals: simple chemistry meets complex physics? Angew Chem Int Ed, 2009, 48: 60-103

3 Jain PK, Huang X, El-Sayed IH, et al. Noble metals on the nanoscale: optical and photothermal properties and some applications in imaging, sensing, biology, and medicine. Acc Chem Res, 2008, 41: 1578-1586

4 Jiang R, Li B, Fang C, et al. Metal/Semiconductor hybrid nanostructures for plasmon-enhanced applications. Adv Mater, 2014, 26: 5274-5309

5 Homola J1, Yee SS, Gauglitz G. Surface plasmon resonance sensors: review. Sens Actuat B-Chem, 1999, 54: 3-15

6 Willets KA, Van Duyne RP. Localized surface plasmon resonance spectroscopy and sensing. Annu Rev Phys Chem, 2007, 58: 267-297

7 Homola J. Surface plasmon resonance sensors for detection of chemical and biological species. Chem Rev, 2008, 108: 462-493

8 Linic S, Christopher P, Ingram DB. Plasmonic-metal nanostructures for efficient conversion of solar to chemical energy. Nat Mater, 2011, 10: 911-921

9 Gilroy KD, Ruditskiy A, Peng HC, et al. Bimetallic nanocrystals: syntheses, properties, and applications. Chem Rev, 2016, 116: 10414-10472

10 Wang D, Li Y. Bimetallic nanocrystals: liquid-phase synthesis and catalytic applications. Adv Mater, 2011, 23: 1044-1060

11 Swearer DF, Zhao H, Zhou L, et al. Heterometallic antennareactor complexes for photocatalysis. Proc Natl Acad Sci USA, 2016, 113: 8916-8920

12 Aslam U, Chavez S, Linic S. Controlling energy flow in multimetallic nanostructures for plasmonic catalysis. Nat Nanotech, 2017, 12: 1000-1005

13 Zhang J, Tang $\mathrm{Y}$, Lee $\mathrm{K}$, et al. Tailoring light-matter-spin interactions in colloidal hetero-nanostructures. Nature, 2010, 466: 91-95

14 Zhang W, Govorov AO, Bryant GW. Semiconductor-metal nanoparticle molecules: hybrid excitons and the nonlinear Fano effect. Phys Rev Lett, 2006, 97: 146804

15 Nan F, Liang S, Liu XL, et al. Sign-reversed and magnitudeenhanced nonlinear absorption of Au-CdS core-shell heteronanorods. Appl Phys Lett, 2013, 102: 163112

16 Sreeramulu V, Haldar KK, Patra A, et al. Nonlinear optical switching and enhanced nonlinear optical response of Au-CdSe heteronanostructures. J Phys Chem C, 2014, 118: 30333-30341

17 Li Y, Pan G, Liu Q, et al. Coupling resonances of surface plasmon in gold nanorod/copper chalcogenide core-shell nanostructures and their enhanced photothermal effect. ChemPhysChem, 2018, 19: $1852-1858$

18 Zou Y, Sun C, Gong W, et al. Morphology-controlled synthesis of hybrid nanocrystals via a selenium-mediated strategy with ligand shielding effect: The case of dual plasmonic $\mathrm{Au}-\mathrm{Cu}_{2-x} \mathrm{Se}$. ACS Nano, 2019, 11: 3776-3785

19 Liu X, Lee $\mathrm{C}$, Law WC, et al. $\mathrm{Au}-\mathrm{Cu}_{2-x} \mathrm{Se}$ heterodimer nanoparticles with broad localized surface plasmon resonance as contrast agents for deep tissue imaging. Nano Lett, 2013, 13: 4333-4339

20 Cao Y, Li S, Chen C, et al. Rattle-type Au@ $\mathrm{Cu}_{2-x} \mathrm{~S}$ hollow mesoporous nanocrystals with enhanced photothermal efficiency for intracellular oncogenic microRNA detection and chemo-photothermal therapy. Biomaterials, 2018, 158: 23-33

21 Lv Q, Min H, Duan DB, et al. Total aqueous synthesis of Au@ 
$\mathrm{Cu}_{2-x} \mathrm{~S}$ core-shell nanoparticles for in vitro and in vivo SERS/PA imaging-guided photothermal cancer therapy. Adv Healthcare Mater, 2018, 8: 1801257

22 Ma L, Chen K, Nan F, et al. Improved hydrogen production of Au-Pt-CdS hetero-nanostructures by efficient plasmon-induced multipathway electron transfer. Adv Funct Mater, 2016, 26: 60766083

23 Ma S, Chen K, Qiu YH, et al. Controlled growth of CdS- $\mathrm{Cu}_{2-x} \mathrm{~S}$ lateral heteroshells on Au nanoparticles with improved photocatalytic activity and photothermal efficiency. J Mater Chem A, 2019, 7: 3408-3414

24 Zhang N, Han C, Fu X, et al. Function-oriented engineering of metal-based nanohybrids for photoredox catalysis: exerting plasmonic effect and beyond. Chem, 2018, 4: 1832-1861

25 Aslam U, Rao VG, Chavez S, et al. Catalytic conversion of solar to chemical energy on plasmonic metal nanostructures. Nat Catal, 2018, 1: 656-665

26 Zhan C, Chen XJ, Yi J, et al. From plasmon-enhanced molecular spectroscopy to plasmon-mediated chemical reactions. Nat Rev Chem, 2018, 2: 216-230

27 Kim D, Resasco J, Yu Y, et al. Synergistic geometric and electronic effects for electrochemical reduction of carbon dioxide using gold-copper bimetallic nanoparticles. Nat Commun, 2014, 5: 4948

28 Xu Z, Lai E, Shao-Horn Y, et al. Compositional dependence of the stability of $\mathrm{AuCu}$ alloy nanoparticles. Chem Commun, 2012, 48: 5626-5628

29 Motl NE, Ewusi-Annan E, Sines IT, et al. Au-Cu alloy nanoparticles with tunable compositions and plasmonic properties: Experimental determination of composition and correlation with theory. J Phys Chem C, 2010, 114: 19263-19269

30 Mallin MP, Murphy CJ. Solution-phase synthesis of sub-10 nm Au-Ag alloy nanoparticles. Nano Lett, 2002, 2: 1235-1237

31 Wang C, Yin H, Chan R, et al. One-Pot synthesis of oleylamine coated AuAg alloy NPs and their catalysis for CO oxidation. Chem Mater, 2009, 21: 433-435

32 Su T, Xiao H, Shen W, et al. Nonlinear size-dependent melting of silica-encapsulated $\mathrm{Ag}-\mathrm{Cu}$ alloy nanoparticles. J Phys Chem C, 2018, 122: 27761-27768

33 Tang W, Peterson AA, Varela AS, et al. The importance of surface morphology in controlling the selectivity of polycrystalline copper for $\mathrm{CO}_{2}$ electroreduction. Phys Chem Chem Phys, 2012, 14: 76-81

34 He R, Wang YC, Wang X, et al. Facile synthesis of pentacle goldcopper alloy nanocrystals and their plasmonic and catalytic properties. Nat Commun, 2014, 5: 4327

35 Zhong Y, Yu X, Fu W, et al. Colorimetric and Raman spectroscopic array for detection of hydrogen peroxide and glucose based on etching the silver shell of Au@Ag core-shell nanoparticles. Microchim Acta, 2019, 186: 802

36 Shan G, Zheng S, Chen S, et al. Detection of label-free $\mathrm{H}_{2} \mathrm{O}_{2}$ based on sensitive Au nanorods as sensor. Colloids Surfs B-Biointerfaces, 2013, 102: 327-330

37 Zhao Y, Zhao J, Shan G, et al. SERS-active liposome@Ag/Au nanocomposite for NIR light-driven drug release. Colloids Surfs B-Biointerfaces, 2017, 154: 150-159

38 Jiang $\mathrm{R}$, Chen $\mathrm{H}$, Shao $\mathrm{L}$, et al. Unraveling the evolution and nature of the plasmons in (Au core)-(Ag shell) nanorods. Adv Mater, 2012, 24: OP200-OP207 preparation of $\mathrm{Au}-\mathrm{Ag}$ core-shell nanorods using anisotropic silver shell formation on gold nanorods. Nanoscale, 2010, 2: 1489-1493

40 Xiang Y, Wu X, Liu D, et al. Gold nanorod-seeded growth of silver nanostructures: from homogeneous coating to anisotropic coating. Langmuir, 2008, 24: 3465-3470

41 Ding S, Yang D, Liu X, et al. Asymmetric growth of Au-core/Agshell nanorods with a strong octupolar plasmon resonance and an efficient second-harmonic generation. Nano Res, 2018, 11: 686695

42 Yang Y, Liu J, Fu ZW, et al. Galvanic replacement-free deposition of $\mathrm{Au}$ on $\mathrm{Ag}$ for core-shell nanocubes with enhanced chemical stability and SERS activity. J Am Chem Soc, 2014, 136: 8153-8156

43 Ma Y, Li W, Cho EC, et al. Au@Ag core-shell nanocubes with finely tuned and well-controlled sizes, shell thicknesses, and optical properties. ACS Nano, 2010, 4: 6725-6734

44 Personick ML, Langille MR, Wu J, et al. Synthesis of gold hexagonal bipyramids directed by planar-twinned silver triangular nanoprisms. J Am Chem Soc, 2013, 135: 3800-3803

45 Murshid N, Gourevich I, Coombs N, et al. Gold plating of silver nanoparticles for superior stability and preserved plasmonic and sensing properties. Chem Commun, 2013, 49: 11355-11357

46 Langille MR, Zhang J, Personick ML, et al. Stepwise evolution of spherical seeds into 20-fold twinned icosahedra. Science, 2012, 337: 954-957

47 Zhang J, Langille MR, Mirkin CA. Photomediated synthesis of silver triangular bipyramids and prisms: the effect of $\mathrm{pH}$ and BSPP. J Am Chem Soc, 2010, 132: 12502-12510

48 Jin R, Charles Cao Y, Hao E, et al. Controlling anisotropic nanoparticle growth through plasmon excitation. Nature, 2003, 425: 487-490

49 Skrabalak SE, Au L, Li X, et al. Facile synthesis of Ag nanocubes and Au nanocages. Nat Protoc, 2007, 2: 2182-2190

$50 \mathrm{Lu}$ X, Au L, McLellan J, et al. Fabrication of cubic nanocages and nanoframes by dealloying $\mathrm{Au} / \mathrm{Ag}$ alloy nanoboxes with an aqueous etchant based on $\mathrm{Fe}\left(\mathrm{NO}_{3}\right)_{3}$ or $\mathrm{NH}_{4} \mathrm{OH}$. Nano Lett, 2007, 7: 1764-1769

51 Chen J, McLellan JM, Siekkinen A, et al. Facile synthesis of goldsilver nanocages with controllable pores on the surface. J Am Chem Soc, 2006, 128: 14776-14777

52 Chen J, Saeki F, Wiley BJ, et al. Gold nanocages: Bioconjugation and their potential use as optical imaging contrast agents. Nano Lett, 2005, 5: 473-477

53 Skrabalak SE, Chen J, Sun Y, et al. Gold nanocages: synthesis, properties, and applications. Acc Chem Res, 2008, 41: 1587-1595

54 Chen J, Glaus C, Laforest R, et al. Gold nanocages as photothermal transducers for cancer treatment. Small, 2010, 6: 811-817

55 Xia Y, Li W, Cobley CM, et al. Gold nanocages: from synthesis to theranostic applications. Acc Chem Res, 2011, 44: 914-924

56 Sun Y, Wiley B, Li ZY, et al. Synthesis and optical properties of nanorattles and multiple-walled nanoshells/nanotubes made of metal alloys. J Am Chem Soc, 2004, 126: 9399-9406

57 Sun Y, Xia Y. Multiple-walled nanotubes made of metals. Adv Mater, 2004, 16: 264-268

58 Seo D, Yoo CI, Chung IS, et al. Shape adjustment between multiply twinned and single-crystalline polyhedral gold nanocrystals: Decahedra, icosahedra, and truncated tetrahedra. J Phys Chem C, 2008, 112: 2469-2475

59 Seo D, Yoo CI, Jung J, et al. Ag-Au-Ag heterometallic nanorods formed through directed anisotropic growth. J Am Chem Soc, 
2008, 130: 2940-2941

60 Yang Y, Wang W, Li X, et al. Controlled growth of Ag/Au bimetallic nanorods through kinetics control. Chem Mater, 2013, 25: 34-41

61 Li C, Sun L, Sun Y, et al. One-pot controllable synthesis of Au@Ag heterogeneous nanorods with highly tunable plasmonic absorption. Chem Mater, 2013, 25: 2580-2590

62 Mayer M, Scarabelli L, March K, et al. Controlled living nanowire growth: precise control over the morphology and optical properties of AgAuAg bimetallic nanowires. Nano Lett, 2015, 15 : 5427-5437

63 Lin S, Lin X, Han S, et al. Width and length dependent SERS performance of core-shell Au@Ag nanorod self-assembled monolayers. J Alloys Compd, 2019, 805: 318-326

64 Zhang C, Zhao H, Zhou L, et al. Al-Pd nanodisk heterodimers as antenna-reactor photocatalysts. Nano Lett, 2016, 16: 6677-6682

65 Li K, Hogan NJ, Kale MJ, et al. Balancing near-field enhancement, absorption, and scattering for effective antenna-reactor plasmonic photocatalysis. Nano Lett, 2017, 17: 3710-3717

66 Wang F, Li C, Chen H, et al. Plasmonic harvesting of light energy for Suzuki coupling reactions. J Am Chem Soc, 2013, 135: 55885601

$67 \mathrm{Qu} \mathrm{Y}$, Cheng R, Su Q, et al. Plasmonic enhancements of photocatalytic activity of $\mathrm{Pt} / \mathrm{n}-\mathrm{Si} / \mathrm{Ag}$ photodiodes using $\mathrm{Au} / \mathrm{Ag}$ core/ shell nanorods. J Am Chem Soc, 2011, 133: 16730-16733

$68 \mathrm{Lu}$ CL, Prasad KS, Wu HL, et al. Au nanocube-directed fabrication of Au-Pd core-shell nanocrystals with tetrahexahedral, concave octahedral, and octahedral structures and their electrocatalytic activity. J Am Chem Soc, 2010, 132: 14546-14553

69 Zhang GR, Wu J, Xu BQ. Syntheses of sub-30 nm Au@Pd concave nanocubes and Pt-on-(Au@Pd) trimetallic nanostructures as highly efficient catalysts for ethanol oxidation. J Phys Chem C, 2012, 116: 20839-20847

70 Li J, Zheng Y, Zeng J, et al. Controlling the size and morphology of Au@Pd core-shell nanocrystals by manipulating the kinetics of seeded growth. Chem Eur J, 2012, 18: 8150-8156

71 Li H, Wu H, Zhai Y, et al. Synthesis of monodisperse plasmonic $\mathrm{Au}$ core-Pt shell concave nanocubes with superior catalytic and electrocatalytic activity. ACS Catal, 2013, 3: 2045-2051

72 Zheng Z, Tachikawa T, Majima T. Single-particle study of Ptmodified Au nanorods for plasmon-enhanced hydrogen generation in visible to near-infrared region. J Am Chem Soc, 2014, 136: 6870-6873

73 Zheng Z, Tachikawa T, Majima T. Plasmon-enhanced formic acid dehydrogenation using anisotropic $\mathrm{Pd}-\mathrm{Au}$ nanorods studied at the single-particle level. J Am Chem Soc, 2015, 137: 948-957

74 Hou S, Hu X, Wen T, et al. Core-shell noble metal nanostructures templated by gold nanorods. Adv Mater, 2013, 25: 3857-3862

75 Zhang $\mathrm{K}, \mathrm{Hu} \mathrm{X}$, Liu J, et al. Formation of PdPt alloy nanodots on gold nanorods: tuning oxidase-like activities via composition. Langmuir, 2011, 27: 2796-2803

76 Sun Y, Wang R, Liu X, et al. Laser-induced formation of $\mathrm{Au} / \mathrm{Pt}$ nanorods with peroxidase mimicking and SERS enhancement properties for application to the colorimetric determination of $\mathrm{H}_{2} \mathrm{O}_{2}$. Microchim Acta, 2018, 185: 445

77 Feng L, Wu X, Ren L, et al. Well-controlled synthesis of Au@Pt nanostructures by gold-nanorod-seeded growth. Chem Eur J, 2008, 14: 9764-9771

78 Grzelczak M, Pérez-Juste J, García de Abajo FJ, et al. Optical properties of platinum-coated gold nanorods. J Phys Chem C,
2007, 111: 6183-6188

79 Wang S, Kristian N, Jiang S, et al. Controlled deposition of Pt on $\mathrm{Au}$ nanorods and their catalytic activity towards formic acid oxidation. Electrochem Commun, 2008, 10: 961-964

80 Grzelczak M, Pérez-Juste J, Rodríguez-González B, et al. Influence of silver ions on the growth mode of platinum on gold nanorods. J Mater Chem, 2006, 16: 3946-3951

81 Khanal BP, Zubarev ER. Polymer-functionalized platinum-ongold bimetallic nanorods. Angew Chem Int Ed, 2009, 48: 68886891

82 Yoo SH, Park S. Platinum-coated, nanoporous gold nanorod arrays: synthesis and characterization. Adv Mater, 2007, 19: 16121615

83 Fennell J, He D, Tanyi AM, et al. A selective blocking method to control the overgrowth of $\mathrm{Pt}$ on Au nanorods. J Am Chem Soc, 2013, 135: 6554-6561

84 Lu Y, Yuan J, Polzer F, et al. In situ growth of catalytic active AuPt bimetallic nanorods in thermoresponsive core-shell microgels. ACS Nano, 2010, 4: 7078-7086

85 Cardinal MF, Mongin D, Crut A, et al. Acoustic vibrations in bimetallic Au@Pd core-shell nanorods. J Phys Chem Lett, 2012, 3: 613-619

86 Su G, Jiang H, Zhu H, et al. Controlled deposition of palladium nanodendrites on the tips of gold nanorods and their enhanced catalytic activity. Nanoscale, 2017, 9: 12494-12502

87 Xiang Y, Wu X, Liu D, et al. Formation of rectangularly shaped $\mathrm{Pd} / \mathrm{Au}$ bimetallic nanorods: Evidence for competing growth of the Pd shell between the $\{110\}$ and $\{100\}$ side facets of Au nanorods. Nano Lett, 2006, 6: 2290-2294

88 Huang J, Zhu Y, Lin M, et al. Site-specific growth of Au-Pd alloy horns on Au nanorods: A platform for highly sensitive monitoring of catalytic reactions by surface enhancement Raman spectroscopy. J Am Chem Soc, 2013, 135: 8552-8561

89 Annan W, Qing P, Yadong L. Rod-shaped Au-Pd core-shell nanostructures. Chem Mater, 2011, 23: 3217-3222

90 Zhang K, Xiang Y, Wu X, et al. Enhanced optical responses of Au@Pd core/shell nanobars. Langmuir, 2009, 25: 1162-1168

91 Lou Z, Fujitsuka M, Majima T. Pt-Au triangular nanoprisms with strong dipole plasmon resonance for hydrogen generation studied by single-particle spectroscopy. ACS Nano, 2016, 10: 6299-6305

92 Jang HJ, Hong S, Park S. Shape-controlled synthesis of Pt nanoframes. J Mater Chem, 2012, 22: 19792-19797

93 Griffin S, Montoni NP, Li G, et al. Imaging energy transfer in Ptdecorated Au nanoprisms via electron energy-loss spectroscopy. J Phys Chem Lett, 2016, 7: 3825-3832

94 Fu QQ, Li HH, Ma SY, et al. A mixed-solvent route to unique $\mathrm{PtAuCu}$ ternary nanotubes templated from $\mathrm{Cu}$ nanowires as efficient dual electrocatalysts. Sci China Mater, 2016, 59: 112-121

95 Zhao F, Yuan Q, Luo B, et al. Surface composition-tunable octahedral $\mathrm{PtCu}$ nanoalloys advance the electrocatalytic performance on methanol and ethanol oxidation. Sci China Mater, 2019, 62: 1877-1887

96 Gangishetty MK, Fontes AM, Malta M, et al. Improving the rates of Pd-catalyzed reactions by exciting the surface plasmons of AuPd bimetallic nanotriangles. RSC Adv, 2017, 7: 40218-40226

97 Ma L, Ding SJ, Yang DJ. Preparation of bimetallic Au/Pt nanotriangles with tunable plasmonic properties and improved photocatalytic activity. Dalton Trans, 2018, 47: 16969-16976

98 Huang Z, Liu S, Zhang Y, et al. Regioselective metal deposition on polymer-Au nanoparticle hybrid chains. Sci China Mater, 2019, 
62: $1363-1367$

99 Aslam U, Linic S. Addressing challenges and scalability in the synthesis of thin uniform metal shells on large metal nanoparticle cores: Case study of Ag-Pt core-shell nanocubes. ACS Appl Mater Interfaces, 2017, 9: 43127-43132

100 Chavez S, Aslam U, Linic S. Design principles for directing energy and energetic charge flow in multicomponent plasmonic nanostructures. ACS Energy Lett, 2018, 3: 1590-1596

101 Rao VG, Aslam U, Linic S. Chemical requirement for extracting energetic charge carriers from plasmonic metal nanoparticles to perform electron-transfer reactions. J Am Chem Soc, 2019, 141: 643-647

102 Li J, Liu J, Yang Y, et al. Bifunctional Ag@Pd-Ag nanocubes for highly sensitive monitoring of catalytic reactions by surface-enhanced Raman spectroscopy. J Am Chem Soc, 2015, 137: 70397042

103 Sun $\mathrm{X}$, Yang X, Zhang Y, et al. Pt-Ag cubic nanocages with wall thickness less than $2 \mathrm{~nm}$ and their enhanced catalytic activity toward oxygen reduction. Nanoscale, 2017, 9: 15107-15114

104 Plana D, Flórez-Montaño J, Celorrio V, et al. Tuning $\mathrm{CO}_{2}$ electroreduction efficiency at $\mathrm{Pd}$ shells on $\mathrm{Au}$ nanocores. Chem Commun, 2013, 49: 10962-10964

105 Yuan X, Zhang L, Li L, et al. Ultrathin Pd-Au shells with controllable alloying degree on Pd nanocubes toward carbon dioxide reduction. J Am Chem Soc, 2019, 141: 4791-4794

106 Lee CW, Yang KD, Nam DH, et al. Defining a materials database for the design of copper binary alloy catalysts for electrochemical $\mathrm{CO}_{2}$ conversion. Adv Mater, 2018, 30: 1704717

$107 \mathrm{Xu} \mathrm{L}$, Wang K, Jiang B, et al. Competitive effect in the growth of Pd-Au-Pd segmental nanorods. Chem Mater, 2016, 28: 73947403

108 Rodal-Cedeira S, Montes-García V, Polavarapu L, et al. Plasmonic Au@Pd nanorods with boosted refractive index susceptibility and SERS efficiency: a multifunctional platform for hydrogen sensing and monitoring of catalytic reactions. Chem Mater, 2016, 28: 9169-9180

109 Liu S, Niu W, Firdoz S, et al. Iodide-switched deposition for the synthesis of segmented Pd-Au-Pd Nanorods: crystal facet matters. Langmuir, 2017, 33: 12254-12259

110 Luo M, Huang H, Choi SI, et al. Facile synthesis of Ag nanorods with no plasmon resonance peak in the visible region by using $\mathrm{Pd}$ decahedra of $16 \mathrm{~nm}$ in size as seeds. ACS Nano, 2015, 9: 1052310532

111 Luo M, Ruditskiy A, Peng HC, et al. Penta-twinned copper nanorods: facile synthesis via seed-mediated growth and their tunable plasmonic properties. Adv Funct Mater, 2016, 26: 12091216

112 Zeng J, Zhu C, Tao J, et al. Controlling the nucleation and growth of silver on palladium nanocubes by manipulating the reaction kinetics. Angew Chem Int Ed, 2012, 51: 2354-2358

113 Zhu C, Zeng J, Tao J, et al. Kinetically controlled overgrowth of $\mathrm{Ag}$ or $\mathrm{Au}$ on Pd nanocrystal seeds: from hybrid dimers to nonconcentric and concentric bimetallic nanocrystals. J Am Chem Soc, 2012, 134: 15822-15831

114 Lim B, Kobayashi H, Yu T, et al. Synthesis of Pd-Au bimetallic nanocrystals via controlled overgrowth. J Am Chem Soc, 2010, 132: 2506-2507

115 Jin $\mathrm{M}$, Zhang $\mathrm{H}$, Wang J, et al. Copper can still be epitaxially deposited on palladium nanocrystals to generate core-shell nanocubes despite their large lattice mismatch. ACS Nano, 2012, 6:
$2566-2573$

116 Zhang J, Tang Y, Lee K, et al. Nonepitaxial growth of hybrid coreshell nanostructures with large lattice mismatches. Science, 2010, 327: 1634-1638

117 Wang JH, Chen M, Luo ZJ, et al. Ceria-coated gold nanorods for plasmon-enhanced near-infrared photocatalytic and photoelectrochemical performances. J Phys Chem C, 2016, 120: 1480514812

$118 \mathrm{Li} \mathrm{B}, \mathrm{Gu} \mathrm{T}$, Ming T, et al. (Gold core)@(ceria shell) nanostructures for plasmon-enhanced catalytic reactions under visible light. ACS Nano, 2014, 8: 8152-8162

119 Li Y, Cain JD, Hanson ED, et al. Au@MoS core-shell heterostructures with strong light-matter interactions. Nano Lett, 2016, 16: 7696-7702

120 Chen K, Ding SJ, Luo ZJ, et al. Largely enhanced photocatalytic activity of $\mathrm{Au} / \mathrm{XS}_{2} / \mathrm{Au}(\mathrm{X}=\mathrm{Re}, \mathrm{Mo})$ antenna-reactor hybrids: charge and energy transfer. Nanoscale, 2018, 10: 4130-4137

121 Li Y, DiStefano JG, Murthy AA, et al. Superior plasmonic photodetectors based on $\mathrm{Au} @ \mathrm{MoS}_{2}$ core-shell heterostructures. ACS Nano, 2017, 11: 10321-10329

122 Sun Z, Yang Z, Zhou J, et al. A general approach to the synthesis of gold-metal sulfide core-shell and heterostructures. Angew Chem Int Ed, 2009, 48: 2881-2885

123 Chen WT, Yang TT, Hsu YJ. Au-CdS core-shell nanocrystals with controllable shell thickness and photoinduced charge separation property. Chem Mater, 2008, 20: 7204-7206

124 Yang TT, Chen WT, Hsu YJ, et al. Interfacial charge carrier dynamics in core-shell Au-CdS nanocrystals. J Phys Chem C, 2010, 114: 11414-11420

125 Chen WT, Lin YK, Yang TT, et al. Au/ZnS core/shell nanocrystals as an efficient anode photocatalyst in direct methanol fuel cells. Chem Commun, 2013, 49: 8486-8488

126 Wang D, Li X, Li H, et al. Semiconductor-noble metal hybrid nanomaterials with controlled structures. J Mater Chem A, 2013, 1: $1587-1590$

127 Ji M, Xu M, Zhang W, et al. Structurally well-defined Au@ $\mathrm{Cu}_{2-x} \mathrm{~S}$ core-shell nanocrystals for improved cancer treatment based on enhanced photothermal efficiency. Adv Mater, 2016, 28: 30943101

128 Ma L, Yang DJ, Luo ZJ, et al. Controlled growth of sulfide on gold nanotriangles with tunable local field distribution and enhanced photocatalytic activity. J Phys Chem C, 2016, 120: 26996-27002

129 Nan F, Liang S, Wang JH, et al. Tunable plasmon enhancement of gold/semiconductor core/shell hetero-nanorods with site-selectively grown shell. Adv Opt Mater, 2014, 2: 679-686

130 Zhu H, Wang Y, Chen C, et al. Monodisperse dual plasmonic $\mathrm{Au} @ \mathrm{Cu}_{2-x} \mathrm{E}(\mathrm{E}=\mathrm{S}, \mathrm{Se})$ core@shell supraparticles: Aqueous fabrication, multimodal imaging, and tumor therapy at in vivo level. ACS Nano, 2017, 11: 8273-8281

131 Zhang L, Blom DA, Wang $\mathrm{H}$. Au- $\mathrm{Cu}_{2} \mathrm{O}$ core-shell nanoparticles: A hybrid metal-semiconductor heteronanostructure with geometrically tunable optical properties. Chem Mater, 2011, 23: 4587-4598

132 Shi X, Ji Y, Hou S, et al. Plasmon enhancement effect in Au gold nanorods@ $\mathrm{Cu}_{2} \mathrm{O}$ core-shell nanostructures and their use in probing defect states. Langmuir, 2015, 31: 1537-1546

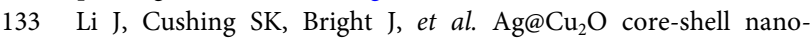
particles as visible-light plasmonic photocatalysts. ACS Catal, 2013, 3: 47-51

134 Meir N, Jen-La Plante I, Flomin K, et al. Studying the chemical, 
optical and catalytic properties of noble metal (Pt, Pd, Ag, Au)$\mathrm{Cu}_{2} \mathrm{O}$ core-shell nanostructures grown via a general approach. J Mater Chem A, 2013, 1: 1763-1769

$135 \mathrm{Yu} \mathrm{H}$, Chen M, Rice PM, et al. Dumbbell-like bifunctional Au$\mathrm{Fe}_{3} \mathrm{O}_{4}$ nanoparticles. Nano Lett, 2005, 5: 379-382

136 Khon E, Mereshchenko A, Tarnovsky AN, et al. Suppression of the plasmon resonance in $\mathrm{Au} / \mathrm{CdS}$ colloidal nanocomposites. Nano Lett, 2011, 11: 1792-1799

137 Haldar KK, Pradhan N, Patra A. Formation of heteroepitaxy in different shapes of Au-CdSe metal-semiconductor hybrid nanostructures. Small, 2013, 9: 3424-3432

138 Yao KX, Liu X, Zhao L, et al. Site-specific growth of Au particles on $\mathrm{ZnO}$ nanopyramids under ultraviolet illumination. Nanoscale, 2011, 3: 4195-4200

$139 \mathrm{Hu}$ D, Diao P, Xu D, et al. Gold/ $/ \mathrm{WO}_{3}$ nanocomposite photoanodes for plasmonic solar water splitting. Nano Res, 2016, 9: $1735-1751$

140 Choi SH, Kim EG, Hyeon T. One-pot synthesis of copper-indium sulfide nanocrystal heterostructures with acorn, bottle, and larva shapes. J Am Chem Soc, 2006, 128: 2520-2521

141 Shaviv E, Banin U. Synergistic effects on second harmonic generation of hybrid CdSe-Au nanoparticles. ACS Nano, 2010, 4: 1529-1538

142 Herring NP, AbouZeid K, Mohamed MB, et al. Formation mechanisms of gold-zinc oxide hexagonal nanopyramids by heterogeneous nucleation using microwave synthesis. Langmuir, 2011, 27: 15146-15154

143 Comin A, Korobchevskaya K, George C, et al. Plasmon bleaching dynamics in colloidal gold-iron oxide nanocrystal heterodimers. Nano Lett, 2012, 12: 921-926

144 Li Y, Zhuang TT, Fan F, et al. Pulsed axial epitaxy of colloidal quantum dots in nanowires enables facet-selective passivation. Nat Commun, 2018, 9: 4947

145 Han SK, Gu C, Zhao S, et al. Precursor triggering synthesis of selfcoupled sulfide polymorphs with enhanced photoelectrochemical properties. J Am Chem Soc, 2016, 138: 12913-12919

146 Zhuang TT, Liu Y, Li Y, et al. Integration of semiconducting sulfides for full-spectrum solar energy absorption and efficient charge separation. Angew Chem Int Ed, 2016, 55: 6396-6400

147 Lee Y, Garcia MA, Frey Huls NA, et al. Synthetic tuning of the catalytic properties of $\mathrm{Au}-\mathrm{Fe}_{3} \mathrm{O}_{4}$ nanoparticles. Angew Chem Int Ed, 2010, 49: 1271-1274

148 Leng C, Zhang X, Xu F, et al. Engineering gold nanorod-copper sulfide heterostructures with enhanced photothermal conversion efficiency and photostability. Small, 2018, 14: 1703077

149 Li P, Wei Z, Wu T, et al. Au-ZnO hybrid nanopyramids and their photocatalytic properties. J Am Chem Soc, 2011, 133: 5660-5663

150 Costi R, Saunders AE, Elmalem E, et al. Visible light-induced charge retention and photocatalysis with hybrid CdSe-Au nanodumbbells. Nano Lett, 2008, 8: 637-641

151 Mokari T, Rothenberg E, Popov I, et al. Selective growth of metal tips onto semiconductor quantum rods and tetrapods. Science, 2004, 304: 1787-1790

152 Mokari T, Sztrum CG, Salant A, et al. Formation of asymmetric one-sided metal-tipped semiconductor nanocrystal dots and rods. Nat Mater, 2005, 4: 855-863

153 Yang J, Elim HI, Zhang QB, et al. Rational synthesis, selfassembly, and optical properties of $\mathrm{PbS}-\mathrm{Au}$ heterogeneous nanostructures via preferential deposition. J Am Chem Soc, 2006, 128: 11921-11926
154 Zeng J, Tao J, Su D, et al. Selective sulfuration at the corner sites of a silver nanocrystal and its use in stabilization of the shape. Nano Lett, 2011, 11: 3010-3015

155 Li M, Yu XF, Liang S, et al. Synthesis of Au-CdS core-shell hetero-nanorods with efficient exciton-plasmon interactions. Adv Funct Mater, 2011, 21: 1788-1794

156 Habas SE, Yang P, Mokari T. Selective growth of metal and binary metal tips on CdS nanorods. J Am Chem Soc, 2008, 130: 32943295

157 Fan FR, Ding Y, Liu DY, et al. Facet-selective epitaxial growth of heterogeneous nanostructures of semiconductor and metal: $\mathrm{ZnO}$ nanorods on Ag nanocrystals. J Am Chem Soc, 2009, 131: 1203612037

158 Kuo CH, Hua TE, Huang MH. Au nanocrystal-directed growth of $\mathrm{Au}-\mathrm{Cu}_{2} \mathrm{O}$ core-shell heterostructures with precise morphological control. J Am Chem Soc, 2009, 131: 17871-17878

159 Chakrabortty S, Yang JA, Tan YM, et al. Asymmetric dumbbells from selective deposition of metals on seeded semiconductor nanorods. Angew Chem Int Ed, 2010, 49: 2888-2892

160 Zeng J, Huang J, Liu C, et al. Gold-based hybrid nanocrystals through heterogeneous nucleation and growth. Adv Mater, 2010, 22: 1936-1940

161 AbouZeid KM, Mohamed MB, El-Shall MS. Hybrid Au-CdSe and Ag-CdSe nanoflowers and core-shell nanocrystals via one-pot heterogeneous nucleation and growth. Small, 2011, 7: 3299-3307

$162 \mathrm{Gu} \mathrm{H}$, Zheng R, Zhang XX, et al. Facile one-pot synthesis of bifunctional heterodimers of nanoparticles: A conjugate of quantum dot and magnetic nanoparticles. J Am Chem Soc, 2004, 126: 5664-5665

163 Peng S, Lei C, Ren Y, et al. Plasmonic/magnetic bifunctional nanoparticles. Angew Chem Int Ed, 2011, 50: 3158-3163

164 Pacholski C, Kornowski A, Weller H. Site-specific photodeposition of silver on $\mathrm{ZnO}$ nanorods. Angew Chem Int Ed, 2004, 43: 4774-4777

165 Zhao Q, Ji M, Qian H, et al. Controlling structural symmetry of a hybrid nanostructure and its effect on efficient photocatalytic hydrogen evolution. Adv Mater, 2014, 26: 1387-1392

166 Liu XD, Chen K, Ma S, et al. Synthesis of Au/CdSe janus nanoparticles with efficient charge transfer for improving photocatalytic hydrogen generation. Nanoscale Res Lett, 2019, 14: 349

167 Liang S, Liu XL, Yang YZ, et al. Symmetric and asymmetric AuAgCdSe hybrid nanorods. Nano Lett, 2012, 12: 5281-5286

168 Wang PF, Chen K, Ma S, et al. Asymmetric synthesis of Au-CdSe core-semishell nanorods for plasmon-enhanced visible-light-driven hydrogen evolution. Nanoscale, 2020, 12: 687-694

169 Liu Y, Hight Walker AR. Facile one-pot synthesis of metalsemiconductor hybrid nanocrystals via chemical transformation: The Case of $\mathrm{Cu}-\mathrm{Cu}_{x} \mathrm{~S}$ heterodimers and hetero-oligomers. J Phys Chem C, 2010, 114: 4264-4271

$170 \mathrm{Wu}$ B, Liu D, Mubeen S, et al. Anisotropic growth of $\mathrm{TiO}_{2}$ onto gold nanorods for plasmon-enhanced hydrogen production from water reduction. J Am Chem Soc, 2016, 138: 1114-1117

171 Jia H, Du A, Zhang H, et al. Site-selective growth of crystalline ceria with oxygen vacancies on gold nanocrystals for near-infrared nitrogen photofixation. J Am Chem Soc, 2019, 141: 50835086

172 Ma L, Yang DJ, Song XP, et al. Pt decorated (Au nanosphere)/ (CuSe ultrathin nanoplate) tangential hybrids for efficient photocatalytic hydrogen generation via dual-plasmon-induced strong Vis-NIR light absorption and interfacial electric field coupling. 
Sol RRL, 2020, 4: 1900376

173 Liu B, Mosa IM, Song W, et al. Unconventional structural and morphological transitions of nanosheets, nanoflakes and nanorods of AuNP@MnO . J Mater Chem A, 2016, 4: 6447-6455

174 Prodan E, Radloff C, Halas NJ, et al. A hybridization model for the plasmon response of complex nanostructures. Science, 2003, 302: 419-422

175 Zhang J, Li W, Li Y, et al. Self-optimizing bifunctional CdS/Cu $\mathrm{Cu}_{2} \mathrm{~S}$ with coexistence of light-reduced $\mathrm{Cu}^{0}$ for highly efficient photocatalytic $\mathrm{H}_{2}$ generation under visible-light irradiation. Appl Catal B-Environ, 2017, 217: 30-36

176 Zhang L, Jing H, Boisvert G, et al. Geometry control and optical tunability of metal-cuprous oxide core-shell nanoparticles. ACS Nano, 2012, 6: 3514-3527

177 Chen $\mathrm{K}, \mathrm{Ma} \mathrm{L}$, Wang JH, et al. Integrating metallic nanoparticles of $\mathrm{Au}$ and $\mathrm{Pt}$ with $\mathrm{MoS}_{2}-\mathrm{CdS}$ hybrids for high-efficient photocatalytic hydrogen generation via plasmon-induced electron and energy transfer. RSC Adv, 2017, 7: 26097-26103

178 Li J, Cushing SK, Zheng P, et al. Solar hydrogen generation by a $\mathrm{CdS}-\mathrm{Au}-\mathrm{TiO}_{2}$ sandwich nanorod array enhanced with $\mathrm{Au}$ nanoparticle as electron relay and plasmonic photosensitizer. J Am Chem Soc, 2014, 136: 8438-8449

179 Guo S, Li X, Zhu J, et al. Au NPs@MoS ${ }_{2}$ sub-micrometer sphere$\mathrm{ZnO}$ nanorod hybrid structures for efficient photocatalytic hydrogen evolution with excellent stability. Small, 2016, 12: 5692-5701

180 Li Y, Wang WN, Zhan Z, et al. Photocatalytic reduction of $\mathrm{CO}_{2}$ with $\mathrm{H}_{2} \mathrm{O}$ on mesoporous silica supported $\mathrm{Cu} / \mathrm{TiO}_{2}$ catalysts. Appl Catal B-Environ, 2010, 100: 386-392

181 Lim DK, Jeon KS, Hwang JH, et al. Highly uniform and reproducible surface-enhanced Raman scattering from DNA-tailorable nanoparticles with 1-nm interior gap. Nat Nanotech, 2011, 6: 452-460

182 Chen Y, Zeng D, Cortie MB, et al. Seed-induced growth of flowerlike Au-Ni-ZnO metal-semiconductor hybrid Nanocrystals for photocatalytic applications. Small, 2015, 11: 1460-1469

183 Shi W, Zeng H, Sahoo Y, et al. A general approach to binary and ternary hybrid nanocrystals. Nano Lett, 2006, 6: 875-881

184 Buck MR, Bondi JF, Schaak RE. A total-synthesis framework for the construction of high-order colloidal hybrid nanoparticles. Nat Chem, 2012, 4: 37-44

185 Chen K, Lin CC, Vela J, et al. Multishell $\mathrm{Au} / \mathrm{Ag} / \mathrm{SiO}_{2}$ nanorods with tunable optical properties as single particle orientation and rotational tracking probes. Anal Chem, 2015, 87: 4096-4099

186 Sharma SP, Thomas III JH. Dielectric breakdown of $\mathrm{Ag}_{2} \mathrm{~S}$ in the Au-Ag 2 S-Ag system. J Appl Phys, 1976, 47: 1808-1811

187 Wu MS, He LJ, Xu JJ, et al. RuSi@Ru(bpy) ${ }_{3}{ }^{2+} / \mathrm{Au} @ \mathrm{Ag}_{2} \mathrm{~S}$ nanoparticles electrochemiluminescence resonance energy transfer system for sensitive DNA detection. Anal Chem, 2014, 86: 45594565

188 Mou NI, Tabib-Azar M. Photoreduction of $\mathrm{Ag}^{+}$in $\mathrm{Ag} / \mathrm{Ag}_{2} \mathrm{~S} / \mathrm{Au}$ memristor. Appl Surf Sci, 2015, 340: 138-142
189 Lombardi A, Grzelczak MP, Crut A, et al. Optical response of individual Au-Ag@SiO 2 heterodimers. ACS Nano, 2013, 7: 25222531

190 Tanaka A, Hashimoto K, Kominami H. Visible-light-induced hydrogen and oxygen formation over $\mathrm{Pt} / \mathrm{Au} / \mathrm{WO}_{3}$ photocatalyst utilizing two types of photoabsorption due to surface plasmon resonance and band-gap excitation. J Am Chem Soc, 2014, 136: 586-589

191 Weng L, Zhang H, Govorov AO, et al. Hierarchical synthesis of non-centrosymmetric hybrid nanostructures and enabled plasmon-driven photocatalysis. Nat Commun, 2014, 5: 4792

192 Mubeen S, Lee J, Singh N, et al. An autonomous photosynthetic device in which all charge carriers derive from surface plasmons. Nat Nanotech, 2013, 8: 247-251

193 Zhuang TT, Liu Y, Sun M, et al. A unique ternary semiconductor(semiconductor/metal) nano-architecture for efficient photocatalytic hydrogen evolution. Angew Chem Int Ed, 2015, 54: 11495-11500

194 Kawawaki T, Nakagawa T, Sakamoto M, et al. Carrier-selective blocking layer synergistically improves the plasmonic enhancement effect. J Am Chem Soc, 2019, 141: 8402-8406

195 Nan F, Xie FM, Liang S, et al. Growth of metal-semiconductor core-multishell nanorods with optimized field confinement and nonlinear enhancement. Nanoscale, 2016, 8: 11969-11975

196 Sanchooli A, Karimipour M, Molaei M. Room temperature synthesis of Au NR@ $\mathrm{Ag}_{2} \mathrm{~S}$ and Au NR@ $\mathrm{Ag}_{2} \mathrm{~S} / \mathrm{CdS}$ core-shells using a facile photochemical approach. Physica E-Low-dimensional Syst NanoStruct, 2019, 109: 133-139

197 Ma L, Liang S, Liu XL, et al. Synthesis of dumbbell-like gold-metal sulfide core-shell nanorods with largely enhanced transverse plasmon resonance in visible region and efficiently improved photocatalytic activity. Adv Funct Mater, 2015, 25: 898-904

198 Feichtner T, Christiansen S, Hecht B. Mode matching for optical antennas. Phys Rev Lett, 2017, 119: 217401

199 Ding SJ, Zhang H, Yang DJ, et al. Magnetic plasmon-enhanced second-harmonic generation on colloidal gold nanocups. Nano Lett, 2019, 19: 2005-2011

Acknowledgements This work was supported by the National Key R\&D Program of China (2017YFA0303402) and the National Natural Science Foundation of China (11874293, 91750113 and 11674254).

Author contributions Wang QQ proposed the outline of the manuscript. Wang QQ, Zhou L, Liang S and Zhong Y designed the figures and wrote the manuscript. Zhong Y, Ma S, Chen K, Wang PF, Qiu YH and Chen Y collected and classified the data. Zhou L finalized and revised the manuscript. All the authors contributed to the general discussion of the paper.

Conflict of interest The authors declare that they have no conflict of interest. 


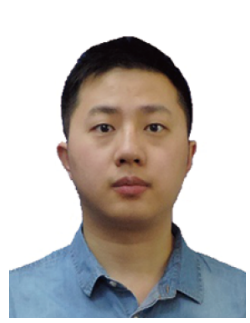

Kai Chen received his BS degree in 2015 from Wuhan University and continued to pursue a $\mathrm{PhD}$ degree in physics under the supervision of Prof. Qu-Quan Wang at Wuhan University. His research interest focuses on plasmonics, 2D materials and their applications for photocatalytic activity.

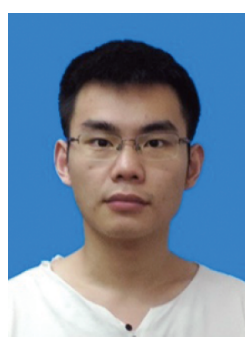

Song Ma received his BSc degree from Wuhan University in 2017 and continued to pursue a $\mathrm{PhD}$ degree in physics under the supervision of Prof. Qu-Quan Wang at Wuhan University. His research interests include plasmonics and photocatalysis.

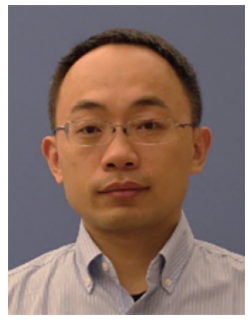

Li Zhou received his BSc, MSc and PhD degrees from Wuhan University. He was a visiting scholar at Nanyang Technological University and Georgia Institute of Technology. He is an associate professor at the School of Physics and Technology, Wuhan University. His research interest includes nanophotonics, plasmonics, functional materials and devices at nanoscale as well as their applications in optical, optoelectronic, energy-related, and biomedical fields.

\section{等离激元异质纳米结构的可控制备及其应用}

钟瑶 $^{1,2 \dagger}$, 马松 ${ }^{1 \dagger}$, 陈凯 $^{3}$, 王鹏飞 ${ }^{1}$, 邱运航 ${ }^{1}$, 梁珊 ${ }^{4}$, 周利 ${ }^{1 *}$, 陈艳伟 ${ }^{2^{*}}$, 王取泉 ${ }^{1,3^{*}}$

摘要 近年来, 具有独特光学性质的等离激元纳米材料研究得到了 广泛关注. 等离激元光学材料与其他功能材料(金属材料或半导体 材料)的结合会产生远胜于单组分材料的性质, 因此在许多光学应 用中展现出优异的性能. 本文综述了具有特定成分、形貌、大小 和结构对称性的等离激元异质纳米结构可控生长的最新进展, 并 介绍了其中等离激元增强的性质和应用性能. 等离激元异质纳米 结构的可控制备和优异性能使其在等离激元增强的非线性光学、 光谱学、光催化、光伏等应用中具有巨大的应用前景. 